\title{
A fixed point theorem for branched covering maps of the plane
}

by

Alexander Blokh and Lex Oversteegen (Birmingham, AL)

\begin{abstract}
It is known that every homeomorphism of the plane which admits an invariant non-separating continuum has a fixed point in the continuum. In this paper we show that any branched covering map of the plane of degree $d,|d| \leq 2$, which has an invariant, non-separating continuum $Y$, either has a fixed point in $Y$, or is such that $Y$ contains a minimal (in the sense of inclusion among invariant continua), fully invariant, non-separating subcontinuum $X$. In the latter case, $f$ has to be of degree -2 and $X$ has exactly three fixed prime ends, one corresponding to an outchannel and the other two to inchannels.
\end{abstract}

1. Introduction. We denote by $\mathbb{C}$ the plane and by $\mathbb{C}^{\infty}$ the Riemann sphere. Homeomorphisms of the plane have been extensively studied. Cartwright and Littlewood [CL51] have shown that each orientation preserving homeomorphism of the plane which has an invariant non-separating subcontinuum $X$ must have a fixed point in $X$. This result was generalized to all homeomorphisms by Bell [Bel78]. The existence of fixed points for orientation preserving homeomorphisms under various conditions was considered in [Bro12, Bro84, Fat87, Fra92, Gui94], and of a point of period two for orientation reversing homeomorphisms in [Bon04].

In this paper we investigate fixed points of light open maps of the plane. By a Theorem of Stoilow [Why42], all such maps have finitely many critical points and are branched covering maps of the plane. In particular, if $\mathcal{C}$ denotes the set of critical points of $f$, then for each $y \in \mathbb{C} \backslash f(\mathcal{C}),\left|f^{-1}(y)\right|$ is finite and independent of $y$. We will denote this number by $d(f)$. All such maps are either positively or negatively oriented (see definitions below); holomorphic maps are prototypes of positively oriented maps. If $f$ is positively oriented then the degree (of the map $f$ ), denoted by degree $(f)$, equals $+d(f)$ and if $f$ is negatively oriented then degree $(f)=-d(f)$. Easy examples, de-

2010 Mathematics Subject Classification: Primary 54H25; Secondary 37C25, 37B45.

Key words and phrases: fixed points, tree-like continuum, branched covering map. 
scribed in Section 2, show that positively and negatively oriented branched covering maps of the plane can be periodic point free.

The following is a well-known open problem in plane topology [Ste35]: Does a continuous function taking a non-separating plane continuum into itself always have a fixed point? Bell announced in 1984 (see also Akis [Aki99]) that the Cartwright-Littlewood Theorem can be extended to holomorphic maps of the plane. This result was extended in [FMOT07] to all branched covering maps (even to all perfect compositions of open and monotone maps) which are positively oriented. Thus, if $f: \mathbb{C} \rightarrow \mathbb{C}$ is positively oriented branched covering map of the plane and $X \subset \mathbb{C}$ is a non-separating continuum such that $f(X) \subset X$ then $X$ contains a fixed point. The main remaining question concerning branched covering maps then is that for negatively oriented maps.

Given a continuum $Y$ in the plane, we denote by $T(Y)$ the topological hull of $Y$, the union of $Y$ and all of the bounded components of $\mathbb{C} \backslash Y$. Also, denote by $U_{\infty}(Y)$ the unbounded component of $\mathbb{C} \backslash Y$. Then $T(Y)=\mathbb{C} \backslash U_{\infty}(Y)$ is a non-separating plane continuum. In this paper we consider a branched covering map $f$ of the plane of degree with absolute value at most 2 and prove the following theorem.

TheOREM 5.2. Suppose that $f: \mathbb{C} \rightarrow \mathbb{C}$ is a branched covering map of degree with absolute value at most 2 and let $Y$ be a continuum such that $f(Y) \subset T(Y)$. Then one of the following holds.

(1) The map $f$ has a fixed point in $T(Y)$.

(2) The continuum $Y$ contains a fully invariant indecomposable continuum $X$ such that $X$ contains no subcontinuum $Z$ with $f(Z) \subset Z$; moreover, in this case degree $(f)=-2$.

It follows that in case $(2) f$ induces a covering map $G$ of the circle of prime ends of $T(X)$ with degree $(G)=-2$ and $T(X)$ has exactly three fixed prime ends and for all of them their principal set is equal to $X$. More precisely, let us consider in the uniformization plane the complement $\mathbb{D}^{\infty}$ to the closed unit disk, and choose a Riemann map $\varphi: \mathbb{D}^{\infty} \rightarrow \mathbb{C}^{\infty} \backslash T(X)$ such that $\varphi(\infty)=\infty$. Then one of the fixed prime ends, say, $\alpha$ corresponds to an outchannel (i.e., for sufficiently small crosscuts $C$ whose preimages in the uniformization plane separate $e^{2 \pi \alpha} \in \mathbb{S}^{1}$ from infinity, $f(C)$ separates $C$ from infinity in $\mathbb{C} \backslash T(X)$ ), and the other two prime ends correspond to inchannels (i.e., for sufficiently small crosscuts $C$ separating the corresponding points on the unit circle from infinity, $C$ separates $f(C)$ from infinity in $\mathbb{C} \backslash T(X)$ ).

Let us outline the main steps of the proof. By known results we may assume that degree $(f)=-2$; we may also assume that $f$ has no fixed points in $T(Y)$. Bell [Bel67] (see also [Sie68, Ili70]) has shown that then $Y$ contains a 
subcontinuum $X$ with the following properties: (1) $X$ is minimal with respect to the property that $f(X) \subset T(X),(2) f(X)=X$ is indecomposable, and (3) there exists an external ray $R$ to $T(X)$ whose principal set is $X$. Let $c$ be the critical point of $f$ and $\tau: \mathbb{C} \rightarrow \mathbb{C}$ be the map such that $\tau(c)=c$ and $\tau(x)$ is the point $y \neq x$ with $f(y)=f(x)$ (if $x \neq c)$. By [Bel78] we assume that $\tau(X) \cap X \neq \emptyset$. By way of contradiction we assume that $X$ is not fully invariant.

The first important step in the proof is made in Lemma 3.7 where we prove that $X \cap \tau(X)$ is a first category subset of $X$. Krasinkiewicz [Kra74] introduced the notions of internal and external composants and described important properties of these objects. His tools are instrumental for the results of Section 3. In Section 4 we construct a modification of the map $f$, which coincides with $f$ on $T(X)$ and for which the external ray $R$ has an invariant tail, i.e. a part of $R$ from some point on to $X$ maps over itself, repelling points away from $X$ in the sense of the order on $R$. In doing so we use a new sufficient condition allowing one to extend a function from the boundary of a domain over the domain. The proof of Theorem 5.2 is given in Section 5. There we study how the ray $R$ approaches $X$ and use the map on $R$ and the fact that $\tau(X) \cap X$ is a first category set in $X$ in order to come up with a sequence of segments of $R$ which map one onto the other and converge to a proper subcontinuum of $X$, a contradiction.

2. Main notions and examples. All maps considered in this paper are continuous. We begin by giving some definitions (avoiding the most standard ones). A map $f: X \rightarrow Y$ is monotone provided for each continuum or singleton $K \subset Y, f^{-1}(K)$ is a continuum or a point. A map $f: X \rightarrow Y$ is light provided for each point $y \in Y, f^{-1}(y)$ is totally disconnected. A map $f: X \rightarrow Y$ is confluent if for each continuum $K \subset Y$ and each component $C$ of $f^{-1}(K), f(C)=K$. It is well known [Why42] that all open maps between compacta are confluent. In the above situation components of $f^{-1}(K)$ are often called pullbacks of $K$.

Every homeomorphism of the plane is either orientation preserving or orientation reversing. In this section we will recall an appropriate extension of this result, which applies to open and perfect maps (see [FMOT07]).

Definition 2.1. Let $f: U \rightarrow \mathbb{C}$ be a map from a simply connected domain $U$ into the plane. Let $S$ be a simple closed curve in $U$, and $p \in$ $U \backslash f^{-1}(f(S))$. Define $f_{p, S}: S \rightarrow \mathbb{S}^{1}$ by

$$
f_{p, S}(x)=\frac{f(x)-f(p)}{|f(x)-f(p)|} .
$$

Then $f_{p, S}$ has a well-defined degree, denoted degree $\left(f_{p, S}\right)$. Note that $\operatorname{degree}\left(f_{p, S}\right)$ is the winding number win $(f, S, f(p))$ of $\left.f\right|_{S}$ about $f(p)$. 
Definition 2.2. A map $f: U \rightarrow \mathbb{C}$ from a simply connected domain $U \subset \mathbb{C}$ is strictly positively oriented (strictly negatively oriented) if for each $p \in T(S) \backslash f^{-1}(f(S))$ we have degree $\left(f_{p, S}\right)>0\left(\operatorname{degree}\left(f_{p, S}\right)<0\right.$ respectively).

Definition 2.3. A map $f: \mathbb{C} \rightarrow \mathbb{C}$ is said to be perfect if preimages of compacta are compacta. A perfect map $f: \mathbb{C} \rightarrow \mathbb{C}$ is oriented provided for each simple closed curve $S$ we have $f(T(S)) \subset T(f(S))$.

REMARK 2.4. Every strictly positively or strictly negatively oriented map is oriented because if a point $p$ is such that $f(p) \notin T(f(S))$ for a simple closed curve $S$, then degree $\left(f_{p, S}\right)=0$. Also, if $Y$ is a continuum then $f(T(Y)) \subset T(f(Y))$ as follows from the definition of an oriented map and continuity arguments.

The following theorem was established in [FMOT07]:

Theorem 2.5. Suppose that $f: \mathbb{C} \rightarrow \mathbb{C}$ is a perfect map. Then the following are equivalent:

(1) $f$ is either strictly positively or strictly negatively oriented.

(2) $f$ is oriented.

(3) $f$ is confluent.

Let us prove a useful lemma related to Theorem 2.5. A branched covering map of the plane is a map $f$ such that at all points, except for finitely many critical points, the map $f$ is a local homeomorphism, at each critical point $c$ the map $f$ acts as $z^{k}$ at 0 for the appropriate $k$, and each point which is not the image of a critical point has the same number $d$ of preimages (then degree $(f)$ equals $d$ if $f$ is positively oriented and $-d$ if $f$ is negatively oriented). By a Theorem of Stoilow [Why42] an open light map of the plane is a branched covering map.

Lemma 2.6. Suppose that $f: \mathbb{C} \rightarrow \mathbb{C}$ is a perfect map such that for every continuum $K$ and every component $K^{\prime}$ of $f^{-1}(K)$ the image $f\left(K^{\prime}\right)$ is not a point. Then $f$ is confluent. If in addition $f$ is light, then it is open (and hence in this case $f$ is a branched covering map).

Proof. Let $f$ be light and show that then it is open. Suppose that $V$ is an open Jordan disk, $x \in V$, and $f(x) \in \operatorname{Bd} f(V)$. Choose a small semiopen arc $I$ in $C \backslash \overline{f(V)}$ with an endpoint of $\bar{I}$ at $f(x)$, and then choose a component $J$ of $f^{-1}(I)$ containing $x$. By the assumptions of the lemma, $J$ is not degenerate. Choose a small disk $V^{\prime}$ so that $x \in V^{\prime} \subset \overline{V^{\prime}} \subset V$. Then the component $J^{\prime}$ of $J \cap \overline{V^{\prime}}$ containing $x$ is not degenerate. Now, the fact that $f$ is light implies that there are points of $J^{\prime}$ mapped into $I \backslash\{f(x)\} \subset C \backslash \overline{f(V)}$, a contradiction. By the Theorem of Stoilow [Why42] then $f$ is a branched covering map of the plane. 
Consider the general case. We can use the so-called monotone-light decomposition. Indeed, consider the map $m$ which collapses all components of sets $f^{-1}(x)$ to points. Then it follows that $f=g \circ m$ where $g$ is a light map. By the above this implies that $f$ is a composition of a monotone map and an open light map of the plane. Clearly, this implies that $f$ is confluent.

A translation by a vector $\mathbf{a}$ (and a translation by a vector a followed by a reflection with respect to an axis non-orthogonal to a), are obvious examples of plane homeomorphisms which are periodic point free. Clearly, any polynomial of degree strictly greater than one, acting on the complex plane, has points of all periods. The following examples show that this is not true for all positively oriented branched covering maps of the plane.

EXAMPLE 2.7. There exists a degree two positively oriented branched covering map of the plane which is periodic point free.

We will use both polar $(r, \theta)$ and rectangular $(x, y)$ coordinates. Set $\varphi(r, \theta)=(r, 2 \theta)$. We will look for a map $f$ in the form $h \circ \varphi$ with $h(x, y)=$ $(x+T(y), y)$ and $T: \mathbb{R} \rightarrow \mathbb{R}$ is a continuous positive function such that $T(s)=T(-s)$. Before we define $T$, let us describe the set $A$ of all points $(r, \theta)$ such that $(r, \theta)$ and $\varphi(r, \theta)$ have the same $y$-coordinates. Then $r \sin (2 \theta)=$ $r \sin (\theta)$. Hence, $\theta \in\{0, \pi / 3, \pi, 5 \pi / 3\}$. So, the set $A$ consists of the $x$-axis and two radial straight lines coming out of $(0,0)$ at angles $\theta= \pm \pi / 3$. Given $s \neq 0$, consider the point $P_{s}$ of intersection between the horizontal line $L_{s}$ of points whose $y$-coordinate is $s$ and the set $A$.

The point $P_{s}$ is the only point on $L_{s}$ with $\varphi$-image also on $L_{s}$. Then the distance between the point $P$ and the point $\varphi\left(P_{s}\right)$ (and the origin) is $2|s| / \sqrt{3}$. Set $T(s)=2|s| / \sqrt{3}+2$. Then $f(x, s) \neq(x, s)$ for any point of $L_{s}$ because all points of $L_{s} \backslash\left\{P_{s}\right\}$ map off $L_{s}$ by $\varphi$, and hence, by the construction, by $f$. On the other hand, $f$ translates $P_{s}$ two units to the right. Hence $L_{s}$ does not contain fixed points. Moreover, since $f(x, 0)=(|x|+2,0), f$ also has no fixed points on the $x$-axis.

To see that $f$ has no periodic points $\left(^{1}\right)$, let $B$ be the set of points in $\mathbb{C}$ whose argument is in $(-\pi / 3, \pi / 3)$. Then $f(\bar{B})=f(\overline{-B}) \subset B$ coincides with the shift of the entire set $\bar{B}$ to the right by two units $(f(\bar{B})=f(\overline{-B})$ because $\varphi(B)=\varphi(-B))$. Let $C=\mathbb{C} \backslash[B \cup-B]$, and let $\operatorname{Im}(z)$ denote the imaginary part of $z$. If $z \in \operatorname{int} C$, then $|\operatorname{Im}(f(z))|<|\operatorname{Im}(z)|$ and if $z \in \operatorname{Bd} C$, then $|\operatorname{Im}(f(z))|=|\operatorname{Im}(z)|$. Let us show that a point $z \in C$ cannot stay in $C$. Indeed, otherwise $y$ has to converge to points of $C$. However, if $y$ were one of these points, then by continuity we would have $|\operatorname{Im}(f(y))|=|\operatorname{Im}(y)|$, which would imply that $y \in \mathrm{Bd} C$ and hence that $f(y) \in B$, contradicting the assumption that $z$ stays in $C$.

$\left({ }^{1}\right)$ We are indebted to M. Misiurewicz for suggesting this argument. 
Hence, for every $z \in \mathbb{C}$, there exists $n$ such that $f^{n}(z) \in B$. Since $f(B)$ $\subset B$, the trajectory stays in $B$ forever. If there exists $m$ such that $f^{m}(z)$ belongs to the real line, then it converges to $+\infty$. To study the orbit of a point $z \in B$ which does not belong to the real line, observe that there exists an increasing function $\xi: \mathbb{R}_{+} \rightarrow \mathbb{R}_{+}$such that if $z \in \overline{f(B)}$ then $|\operatorname{Im}(f(z))| \geq|\operatorname{Im}(z)|+\xi(|\operatorname{Im}(z)|)$. Therefore if $z \in \overline{f(B)}$ does not belong to the real line then $\left|\operatorname{Im}\left(f^{k}(z)\right)\right| \rightarrow \infty$ as $k \rightarrow \infty$. Hence in fact $\left|f^{k}(z)\right| \rightarrow \infty$ for any point $z$ and $f$ has no periodic points.

The example above can be easily modified to obtain a periodic point free branched covering map of degree -2 .

3. Basic preliminaries. A continuum $X$ is called indecomposable if $X$ cannot be written as the union of two proper subcontinua. Also, $Z$ is unshielded if $Z=\operatorname{Bd} U_{\infty}(Z)$. We argue by way of contradiction, therefore the following is our main assumption.

Main Assumption. The map $f: \mathbb{C} \rightarrow \mathbb{C}$ is a branched covering map and $Y \subset \mathbb{C}$ is a continuum such that $f(Y) \subset T(Y)$ and $\left.f\right|_{T(Y)}$ is fixed point free.

Bell [Bel67] has shown that in this case $Y$ contains a subcontinuum $X$ which is minimal with respect to the property that $f(X) \subset T(X)$ (then, clearly, $f(X) \subset T(X)$ ) and which must have the following properties (see [Sie68, Ili70] for alternative proofs):

(A0) $X$ is minimal among continua $Z \subset Y$ such that $f(Z) \subset T(Z)$;

(A1) $f(X)=X$ and $T(X)$ is fixed point free;

(A2) there exists a curve $R_{\beta}$ (a conformal external ray, see below) in $U_{\infty}(Z)$ such that $X=\overline{R_{\beta}} \backslash R_{\beta}$ (so that $X$ is unshielded and has empty interior);

(A3) $X$ is indecomposable.

We will use $X$ exclusively for a continuum with the just listed properties (A0)-(A3) which are ingredients of the standing assumption on $X$. Our main aim is to show that then $X$ is fully invariant (i.e., $f^{-1}(X)=X$ ). Thus, by way of contradiction we can add the following to our standing assumption (as we progress, the standing assumption will be augmented by other ingredients as well).

(A4) The set $X$ is not fully invariant.

Below we list well-known facts from Carathéodory theory. Good sources are the books [Mil00] and [Pom92]. Let $\mathbb{D}$ be the open unit disk in the complex plane and $\mathbb{D}^{\infty}=\mathbb{C}^{\infty} \backslash \overline{\mathbb{D}}$. Let $\varphi: \mathbb{D}^{\infty} \rightarrow \mathbb{C}^{\infty} \backslash T(X)$ be a conformal map such that $\varphi(\infty)=\infty$. An external ray $R_{\alpha}=\varphi\left(\left\{r e^{2 \pi i \alpha} \mid r>1\right\}\right)$ is the $\varphi$-image of the radial line segment $r_{\alpha}=\left\{r e^{2 \pi i \alpha} \mid r>1\right\}$. Clearly, an 
external ray is diffeomorphic to the positive real axis. If $R$ is an external ray and $x$ is a point such that $\bar{R} \backslash R=\{x\}$ then $R$ is said to land on $x$. For convenience we extend the map $\varphi$ onto all angles whose rays land: if the ray $R_{\alpha}$ lands at a point $x$, we set $\varphi\left(e^{2 \pi i \alpha}\right)=x$. Observe that the extended map $\varphi$ is not necessarily continuous at angles whose rays land. Still, this extension is convenient and will be used in what follows.

A crosscut $C$ (of $T(X)$ or of $U_{\infty}(X)$ ) is an open arc in $\mathbb{C} \backslash T(X)$ whose closure is a closed arc with its endpoints in $T(X)$. If $C$ is a crosscut, then by the shadow of $C$, denoted $\operatorname{Sh}(C)$, we mean the bounded component of $\mathbb{C}^{\infty} \backslash[T(X) \cup C]$. Sometimes the crosscut which gives rise to a shadow is said to be the gate of the shadow. In the uniformization plane we consider $\overline{\mathbb{D}}$ as a continuum analogous to $X$, which allows us to talk about crosscuts of $\mathbb{D}^{\infty}$ too. Moreover, given a crosscut in $\mathbb{D}^{\infty}$ we can then talk about its shadow etc. It is known that if $C$ is a crosscut of $T(X)$, then $\varphi^{-1}(C)$ is a crosscut of $\mathrm{Bd} \mathbb{D}^{\infty}$, and $\varphi^{-1}(\operatorname{Sh}(C))=\operatorname{Sh}\left(\varphi^{-1}(C)\right)$.

We say that a crosscut $C$ is an $R_{\alpha}$-essential crosscut if $R_{\alpha} \cap C$ is a single point, called the central point, and the intersection of $C$ and $R_{\alpha}$ is transverse. A sequence of crosscuts $\left\{C_{i}\right\}$ of $T(X)$ is a fundamental chain provided $C_{i+1} \subset \operatorname{Sh}\left(C_{i}\right), \overline{C_{i+1}} \cap \overline{C_{i}}=\emptyset$ for each $i$, and $\lim \operatorname{diam}\left(C_{i}\right)=0$. Two fundamental chains $Q=\left\{q_{n}\right\}$ and $Q^{\prime}=\left\{q_{n}^{\prime}\right\}$ are said to be equivalent if $\operatorname{Sh}\left(q_{n}\right)$ contains all but finitely many crosscuts $q_{n}^{\prime}$, and $\operatorname{Sh}\left(q_{n}^{\prime}\right)$ contains all but finitely many crosscuts $q_{n}$. A prime end of $U_{\infty}(X)$ is an equivalence class of fundamental chains; a fundamental chain is said to belong to its prime end.

Given a fundamental chain $\left\{C_{i}\right\}$, the set $\lim \varphi^{-1}\left(C_{i}\right)$ is a point $e^{2 \pi i \alpha} \in$ $\mathrm{Bd} \mathbb{D}^{\infty}, \alpha \in[0,1)$; the corresponding prime end may then be identified with the angle $\alpha$. Given a prime end $\alpha$ and a corresponding fundamental chain $\left\{C_{i}\right\}$, denote by $\operatorname{Imp}(\alpha)$, called the impression of $\alpha$, the set $\bigcap \overline{\operatorname{Sh}\left(C_{i}\right)}$; it is known that $\operatorname{Imp}(\alpha)$ does not depend on the choice of a fundamental chain and therefore is well-defined. Also, consider the set $\Pi(\alpha)=\overline{R_{\alpha}} \backslash R_{\alpha}$, called the principal set of $R_{\alpha}$ (or just of $\alpha$ ). It is known that $\Pi(\alpha) \subset \operatorname{Imp}(\alpha)$ and that for each point $x \in \Pi(\alpha)$ there exists a fundamental chain $C_{i}$ of the prime end $\alpha$ such that $C_{i} \rightarrow x$.

The last claim can be improved a little. It was shown in [BO06] that given an angle $\alpha$, there exists for each $z \in \mathbb{R}_{\alpha}$ an $R_{\alpha}$-essential crosscut $C_{z}$ such that $\lim \operatorname{diam} C_{z}=0$ as $z \rightarrow X$. We call such a family $C_{z}$ a defining family of crosscuts of the prime end $\alpha$. For convenience we order each $R_{\alpha}$ so that $x<_{\alpha} y$ if and only if the subarc of $R_{\alpha}$ from $y$ to $\infty$ is contained in the subarc of $R_{\alpha}$ from $x$ to $\infty$ (thus, as the points move along $R_{\alpha}$ from infinity towards $X$, they decrease in the sense of the order on $R_{\alpha}$ ). Denote by $(a, b)_{\alpha}$ the set of points in $R_{\alpha}$ enclosed between the points $a, b \in R_{\alpha}$. 
Also, set $(0, a)_{\alpha}=\left\{x \in R_{\alpha} \mid x<_{\alpha} a\right\}$. Similarly we define semi-open and closed subsegments of $R_{\alpha}$ when possible (e.g., if $R_{\alpha}$ lands, it makes sense to talk of $[0, a]_{\alpha}$, but otherwise the set $[0, a]_{\alpha}$ is not defined). Also, similarly we define relations $\leq_{\alpha},>_{\alpha}$ and $\geq_{\alpha}$. By a tail of $R_{\alpha}$ we mean the set of all points $y \in R_{\alpha}$ such that $y<_{\alpha} z$ (or $y \leq_{\alpha} z$ ) for some $z \in R_{\alpha}$.

It is well known that the geometry of the ray $R_{\beta}$ in (A2) and of the continuum $X$ is quite complicated. The ray approaches $X$ so that on either side of $R_{\beta}$ the distance to $X$ goes to 0 while it simultaneously accumulates upon the entire $X$. It then follows from properties of conformal maps that round balls, disjoint from $X$ but not from $R_{\beta}$, with points of the intersection with $R_{\beta}$ approaching $X$ must go to 0 in diameter. One can say that $R_{\beta}$ "digs a dense channel" in the plane eventually accumulating on $X$ by (A2).

As was explained in the Introduction, the main remaining question concerning the fixed point problem for branched covering maps is that dealing with negatively oriented maps of degree $d \leq-2$. Since we are interested in maps $f$ such that $|\operatorname{degree}(f)| \leq 2$ we may make the following assumption.

(A5) From now on we assume that $f$ is of degree -2 .

Then $f$ has a unique critical point, denoted by $c$, and a unique critical value, denoted by $v=f(c)$. Let $\tau: \mathbb{C} \rightarrow \mathbb{C}$ be the involution defined by $\tau(c)=c$ and if $x \neq c, \tau(x)=x^{\prime}$ where $\left\{x^{\prime}\right\}=f^{-1}(f(x)) \backslash\{x\}$. Clearly, $\tau^{2}=$ id (i.e., the map $\tau$ is an idempotent homeomorphism of the plane); sometimes we call $\tau(z)$ the sibling of $z$. Let us establish basic properties of $f$ in the following lemma (some of the properties hold in more general situations, but we do not need such generality in this paper).

Lemma 3.1. The following facts hold.

(1) If $Z$ is a continuum then $f(\operatorname{int}(T(Z))) \subset \operatorname{int}(T(f(Z))$.

(2) Suppose that $K$ is a non-separating continuum. If $v \notin K$ then there are exactly two pullbacks of $K$ which are disjoint and map onto $K$ homeomorphically (on their neighborhoods). If $v \in K$ then $f^{-1}(K)$ is the unique pullback of $K$ which must contain $c$.

(3) If $Y$ is a continuum and $C$ is a pullback of $Y$ then $T(C)$ is a pullback of $T(Y)$ (and hence $f(T(C))=T(Y)$ ). In particular, a pullback of a non-separating continuum is non-separating.

(4) Suppose that $U$ is a simply connected domain such that $f(U)$ is also simply connected. If $\left.f\right|_{U}$ is not a homeomorphism then $U$ must contain a critical point.

Proof. (1) Suppose otherwise. Then there is a point $x \in \operatorname{int}(T(Z))$ such that $f(x) \notin \operatorname{int}(T(f(Z)))$. By Remark 2.4, $f(x) \in T(f(Z))$. Since $f$ is open, we can then find a point $y \in \operatorname{int}(T(Z))$ such that $f(y)$ is outside $T(f(Z))$, contradicting Remark 2.4. 
(2) If $v \notin K$ we can take a curve $Q$ from $v$ to infinity disjoint from $K$. Then $f^{-1}(Q)$ is a curve which cuts $\mathbb{C}$ into two open half-planes and is disjoint from $f^{-1}(K)$. Also, each half-plane maps onto $\mathbb{C} \backslash K$ homeomorphically. Thus, in this case $f^{-1}(K)$ consists of two components each of which maps onto $K$ homeomorphically (on sufficiently small neighborhoods of the pullbacks). Suppose that $v \in K$. Then $f^{-1}(K)$ cannot have more than one component because $f$ is confluent (hence each pullback of $K$ maps onto $K$ ) and $v$ has a unique preimage $c$.

(3) Let us apply (1) to $T(Y)$. If $v \notin T(Y)$ then $C$ must be a homeomorphic pullback of $Y$, which implies the desired. Let $v \in T(Y)$ and set $Z=f^{-1}(T(Y))$ where by (1), $Z$ is the unique pullback of $T(Y)$. Let us show that $C$ is then the unique pullback of $Y$. Let $Y^{\prime}$ be the boundary of $U_{\infty}(T(Y))$, and $Z^{\prime}$ be the boundary of $U_{\infty}(Z)$. It follows that $f\left(Z^{\prime}\right) \subset Y^{\prime}$ $\subset Y$. On the other hand, by (1) no point of $\operatorname{int}(Z)$ can map to a point of $Y^{\prime}$, and by the construction no point from $\mathbb{C} \backslash Z$ can map to a point of $Y^{\prime}$. Hence $Z^{\prime}=f^{-1}\left(Y^{\prime}\right)$.

This implies that $f^{-1}(Y)$ is connected. Indeed, suppose otherwise. Then there are two pullbacks $Y_{1}, Y_{2}$ of $Y$ each of which maps onto $Y$. This implies that $Z^{\prime}=\left(Y_{1} \cap Z^{\prime}\right) \cup\left(Y_{2} \cap Z^{\prime}\right)$, which contradicts the fact that $Z^{\prime}$ is a continuum. Thus, $C=f^{-1}(Y)$. Moreover, $Z^{\prime} \subset C$ and hence $T\left(Z^{\prime}\right)=$ $Z \subset T(C)$. On the other hand, by Remark 2.4, $f(T(C)) \subset T(Y)$ and so $T(C) \subset Z$. Hence $T(C)=Z$. If $Y$ is non-separating, then $T(Y)=Y$. By the above $T(C)$ is a pullback of $T(Y)=Y$ containing $C$, that is, $C$. Thus, $C$ is non-separating as desired.

(4) It immediately follows from (2) that $c \in \bar{U}$ and $v \in f(\bar{U})$. However, we need to show that $c \in U$. Take $x, y \in U$ such that $f(x)=f(y)=z$ and connect them with an arc $I \subset U$. Then $f(I) \subset f(U)=V$. Since $V$ is simply connected by the assumption of the lemma, $T(f(I)) \subset V$. Since $f(x)=f(y)$, it follows that $J=f^{-1}(f(I))$ is the unique pullback of $f(I)$ (because $f$ is confluent and both preimages of $z$ belong to $I$ ). By (2) and (3), $T(J)$ is the unique pullback of $T(f(I))$, and $v \in T(f(I))$. Since $T(f(I)) \subset V=f(U)$, the unique preimage $c$ of $v$ belongs to $U$ as desired.

Suppose that $v \notin T(X)$. Then by Lemma 3.1 it follows that there exists a neighborhood $U$ of $T(X)$ on which $f$ is a homeomorphism. Again, by Bell's results [Bel78] this implies existence of an $f$-fixed point $x \in T(X)$. (Alternatively, the proof given below in Section 5 can easily be adapted to cover this case.) Therefore we extend our standing assumption as follows.

(A6) From now on we assume that $v \in T(X)$.

Since $f$ is oriented and $f(X)=X, f(T(X)) \subset T(f(X))=T(X)$. By Lemma 3.1 the continuum $\widehat{X}=f^{-1}(T(X)) \supset T(X)$ is non-separating and 
maps onto $T(X)$ in a two-to-one fashion (except for the point $c$ ). Let us list simple consequences of our standing assumption as applies to $X$ in this case.

Lemma 3.2. The set $T(X)$ is not fully invariant, $\tau(X) \not \subset T(X)$, and $X \cap \tau(X) \neq \emptyset$.

Proof. Let us show that $T(X)$ is not fully invariant. By Lemma 3.1 no point from the interior of $T(X)$ can map to $X$ (recall that $X$ is unshielded by (A2) and hence no point of $X$ belongs to $\operatorname{int}(T(X))$. Hence if $T(X)$ is fully invariant then so is $X$, contradicting (A4). This implies that there are points of $\tau(T(X))$ outside $T(X)$, and hence $\tau(X)=\mathrm{Bd} \tau(T(X))$ cannot be contained in $T(X)$.

Finally, suppose that $X \cap \tau(X)=\emptyset$. Since there are points of $\tau(X)$ outside $T(X)$, this implies that $\tau(T(X))=T(\tau(X))$ is disjoint from $T(X)$. Hence $\left.f\right|_{T(X)}$ is a homeomorphism and so $f(T(X))=T(X)$. However, then by (A6) we have $c \in T(X)$, a contradiction with $T(X)$ and $\tau(T(X))$ being disjoint. We conclude that $X \cap \tau(X) \neq \emptyset$ as desired.

For convenience let us make the conclusions of Lemma 3.2 a part of our standing assumption.

(A7) $T(X)$ is not fully invariant, $\tau(X) \not \subset T(X)$, and $X \cap \tau(X) \neq \emptyset$.

A composant of $x$ in a continuum $Y$ is the union of all proper subcontinua of $Y$ which contain $x$. If $Y$ is indecomposable then any two composants of $Y$ are either equal or disjoint; clearly, if $g(Y) \subset Y$ for some continuous map $g$, then the image of a composant, being a connected set, either coincides with $Y$, or is contained in a composant of $Y$. It follows from the definition that if $Z$ is a composant of $Y$ then for each $p, q \in Y$, there exists a subcontinuum $P \subset Y$ such that $p, q \in P$. It is well known [Kra74] that if $Y$ is indecomposable, then each composant in a dense first category $F_{\sigma}$-subset of $Y$. By the Baire Category Theorem there are uncountably many distinct composants in an indecomposable continuum.

Again, assume that $Y$ is indecomposable. A composant $Z$ of $Y$ is internal if every continuum $L \subset \mathbb{C}$ which meets $\mathbb{C} \backslash Y$ and $Z$, intersects all composants of $Y$. Equivalently, a composant $Z$ of $Y$ is internal if and only if every continuum $L \subset \mathbb{C}$ which meets $\mathbb{C} \backslash Z$ and $Z$, intersects all composants of $Y$ (indeed, if $C \subset Y$ meets $Z$ and $Y \backslash Z$ then $C$ must coincide with $Y$ ). A composant which is not internal is called external. We denote the union of all external composants by $E_{Y}^{*}$. Clearly, an internal composant $Z$ does not contain accessible points: if $z \in Z$ is accessible (from $\mathbb{C} \backslash Y$ ), then we can choose an arc in the appropriate component of $\mathbb{C} \backslash Y$ with an endpoint at $z$ which intersects $Y$ only at $z$, a contradiction with $Z$ being internal. Thus, an external composant is a generalization of a composant containing an accessible (from $\mathbb{C} \backslash Y$ ) point. 
The following results are due to Krasinkiewicz [Kra74].

Lemma 3.3 (Krasinkiewicz). Let $Y$ be an indecomposable continuum in the plane. Then the following claims hold.

(1) The set $E_{Y}^{*}$ is a first category $F_{\sigma}$-subset of $Y$; hence, the union of all internal composants is a dense $G_{\delta}$-set in $Y$.

(2) Let $C$ be an internal composant of $Y$. If $L$ is any continuum which meets $C$ and the complement of $Y$, and does not contain $Y$, then there exists a neighborhood $U$ of $L$ and a continuum $Z \subset C$ which separates $U$ between two distinct points of $L$.

A plane continuum is called tree-like if it is one-dimensional (has no interior in the plane) and non-separating. Recall that $X$ is an invariant continuum which is minimal among continua with respect to the property that $f(X) \subset T(X)$. The set $X$ has a number of properties listed at the beginning of this section, in particular it is indecomposable (and hence onedimensional) and unshielded. We now study other properties of $X$. First we need a few technical lemmas.

Lemma 3.4. Suppose that $A \subset X$ is a dense $G_{\delta}$-subset of $X$. Then $f(A)$ is not a first category subset of $X$.

Proof. First let us show that there exists a point $x \in X$ and a small neighborhood $U$ of $x$ such that $\left.f\right|_{U}$ is a homeomorphism onto its image and $f(X \cap U)=f(U) \cap X$. Indeed, this is obvious if $X$ is fully invariant. Otherwise choose a point $x \in X$ so that $\tau(x) \notin X$ (i.e., $f(x)$ has a unique preimage in $X$, namely $x$, and $x$ is not critical). If now $U$ is a sufficiently small neighborhood of $x$, then $\tau(U) \cap X=\emptyset$; hence $f(U) \cap X$ consists of points of $X$ which cannot have preimages in $\tau(U) \cap X=\emptyset$, but must have some preimages because $f(X)=X$. The only preimage points of $f(U) \cap X$ are those in $U \cap X$, which implies that $f(X \cap U)=f(U) \cap X$ as desired.

Now, by the conditions of the lemma $A \cap(X \cap \bar{U})$ is a $G_{\delta}$-subset of $X \cap \bar{U}$, hence by the previous paragraph $f(A \cap(X \cap \bar{U}))$ is a $G_{\delta}$-subset of $X \cap f(U)$. Therefore $f(A)$ cannot be a first category subset of $X$.

The next lemma uses Lemma 3.4.

Lemma 3.5. Suppose that $D$ is a union of some composants of $X$ which is a first category subset of $X$. Then there exists a composant $T$ of $X$ such that $f(T) \cap D=\emptyset$.

Proof. By way of contradiction suppose that for every composant $T$ the image $f(T)$ of $T$ intersects $D$. Take a composant $Q$ whose image contains points not from $D$. By the assumption $Q$ also has points mapped into $D$. Hence $f(Q)$ contains points of at least two distinct composants, which implies that $f(Q)=X$. If there is another composant $R$ such that $f(R)$ is not 
contained in $D$, then it again follows that $f(R)=X$. Since $f$ is two-to-one, this implies that $X=Q \cup R$, which is impossible because there are countably many pairwise disjoint composants of $X$. Hence for any composant $R \neq Q$ we have $f(Q) \subset D$. However, the union of all composants except for $Q$ is a $G_{\delta}$-subset of $X$ while the set $D$ is a first category subset of $X$. By Lemma 3.4 this is impossible.

The next lemma studies the images of composants.

Lemma 3.6. Suppose that $Z$ is an internal composant of $X$. Then $f(Z)$ is an internal composant of $X$.

Proof. By way of contradiction suppose that $f(Z)$ is not an internal composant of $X$. Then there are two possibilities: (1) $f(Z)$ is an external composant of $X$ or $f(Z)=X$, or $(2) f(Z)$ is contained in a composant with which it does not coincide. We consider these possibilities separately.

(1) Suppose that $f(Z)$ is either an external composant or the entire $X$. Then there exists a continuum $B$ such that $B \cap f(Z) \neq \emptyset \neq B \backslash X$ and $B \cap X$ is contained in the union of some, but not all, composants of $X$. Then all composants which intersect $B$ are external, and hence their union $D$ is a first category subset of $X$.

Choose $z \in B \cap f(Z)$ and then $z^{\prime} \in Z$ such that $f\left(z^{\prime}\right)=z$. Then choose the pullback $B^{\prime}$ of $B$ which contains $z^{\prime}$. Since $Z$ is an internal composant and $B^{\prime}$ meets $\mathbb{C} \backslash X$, by definition $B^{\prime}$ intersects all composants of $X$. Hence all composants of $X$ have points mapped into the union $D$ of some composants which is a first category subset of $X$. By Lemma 3.5, this is impossible.

(2) Suppose that $f(Z) \subsetneq Y$ where $Y$ is a composant of $X$. Choose a subcontinuum $E \subset Y$ which contains a point $z \in f(Z)$ and a point of $y \in Y \backslash$ $f(Z)$. Choose the pullback $E^{\prime}$ of $E$ which contains a point $z^{\prime} \in Z$ such that $f\left(z^{\prime}\right)=z$. Then $E^{\prime} \not \subset X$ because otherwise it would be contained in $Z$ and its image would not contain $y$. Hence $E^{\prime}$ meets $\mathbb{C} \backslash X$ and contains the point $z^{\prime} \in Z$, which implies that $E^{\prime}$ intersects all composants of $X$ (because $Z$ is an internal composant). Since $f\left(E^{\prime}\right)=E$, this implies that all composants of $X$ have points mapped into $Y$, and $Y$, being a composant of $X$, is a first category subset of $X$. Again, by Lemma 3.5 this is impossible.

In what follows we will use the following lemma which studies the set $\tau(X) \cap X$. In the proof we rely upon the above developed tools.

Lemma 3.7. The set $\tau(X) \cap X$ is contained in the union $E_{X}^{*}$ of all external composants of $X$. In particular, $\tau(X) \cap X$ is a proper closed subset of $X$ with empty interior in $X$.

Proof. By (A7), $\tau(X) \cap X \neq \emptyset$ and $\tau(X) \backslash X \neq \emptyset$. Choose $x \in \tau(X) \backslash X$, then $\tau(x) \in X \backslash \tau(X)$, and hence $\tau(X) \cap X$ is a proper, closed subset of $X$. The fact that $\tau(X) \cap X$ has empty interior in $X$ is much less trivial. 
Let us first show that at most countably many composants of $X$ contain a subcontinuum which separates $\mathbb{C}$. Indeed, if $C \subset Z$ is a separating continuum, we can associate to $Z$ a bounded component $V_{Z}$ of $\mathbb{C} \backslash C$. Since $X$ is unshielded, the sets $V_{Z}, V_{Q}$ for distinct composants $Z, Q$ of $X$ are disjoint. Hence at most countably many composants of $X$ contain a subcontinuum which separates $\mathbb{C}$. Also, there is exactly one composant which contains the critical value $v$. By Lemma 3.3(1) and since each composant is a first category $F_{\sigma}$-subset of $X$ (see Lemma 2.1 of [Kra74]), the union of the above listed countably many composants and all external composants of $X$ is still a first category $F_{\sigma}$-subset of $X$. Its complement is the union $I_{X}^{*}$ of points of all composants from the collection $I_{X}$ of all internal composants of $X$ for which every subcontinuum is tree-like not containing the critical value $v$. Thus, $I_{X}^{*}$ is still a dense $G_{\delta^{-}}$subset of $X$.

By Theorem 4.2 of $[\mathrm{CMT}], f\left(E_{X}^{*}\right)$ is a first category $F_{\sigma}$-subset of $X$. Hence we can choose a point $y \in I_{X}^{*} \backslash f\left(E_{X}^{*}\right)$. Let $Y$ be the internal composant of $X$ which contains $y$. Choose $z \in f^{-1}(y) \cap X$; then $z$ is contained in an internal composant $Z$ of $X$. By Theorem 5.5 of [Rog98], $f^{-1}(Y)=Y_{1} \cup Y_{2}$ such that $Y_{1} \cap Y_{2}=\emptyset$ and for each $i$ and any $p, q \in Y_{i}$, there exists a subcontinuum $P \subset Y_{i}$ such that $p, q \in P$. Moreover, the map $\left.f\right|_{Y_{i}}: Y_{i} \rightarrow Y$ is a bijection for each $i$. Assume that $z \in Y_{1}$. Then by Lemma 3.6, $f(Z)=Y$ and $Z=Y_{1}$. Hence $X$ and $\tau(X)$ contain the disjoint, internal composants $Y_{1}$ and $\tau\left(Y_{1}\right)=Y_{2}$, respectively.

Let us show that if $Q$ is any internal composant of $X$, then $Q \cap Y_{2}=\emptyset$. Indeed, suppose otherwise. Then by symmetry the internal composant $\tau(Q)$ of $\tau(X)$ intersects $Y_{1}$. Choose a point $u \in \tau(Q) \backslash X$ and a point $v \in \tau(Q) \cap Y_{1}$; then choose a continuum $L \subset \tau(Q)$ which contains both $u$ and $v$. Since $Y_{1}$ is an internal composant of $X$, this implies that $\tau(Q)$ intersects all composants of $X$. On the other hand, $f(\tau(Q))=f(Q)$ is an internal composant of $X$ by Lemma 3.6. Thus, the images of all composants of $X$ are non-disjoint from the composant $f(Q)$, contradicting Lemma 3.5.

In order to finish the proof it suffices to show that $\tau(X) \cap X \subset E_{X}^{*}$. Suppose that this is not the case. Then $\tau(X)$ meets an internal composant $I$ of $X$. It is easy to verify that Lemma 3.3 applies to this situation with $\tau(X)$ playing the role of $L, I$ playing the role of $C$, and $X$ playing the role of $Y$. Thus, it follows from Lemma 3.3(2) that there exists a neighborhood $U$ of $\tau(X)$ and a continuum $K \subset I$ such that $K$ separates two points of $\tau(X)$ in $U$. Since by the above $Y_{2}$ and $I$ are disjoint, $Y_{2}$ is contained in one component of $U \backslash K$, contradicting the fact that $Y_{2}$ is dense in $\tau(X)$. This completes the proof of the lemma.

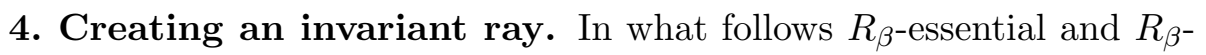
defining crosscuts are called simply essential and defining. To begin with, 
we need a lemma which will allow us to simplify the applications of results of [FMOT07, OT08, KP94] in this section. For simplicity when talking of angles we often mean the points of $\mathbb{S}^{1}$ with arguments equal to these angles; here $\mathbb{S}^{1}$ is considered as the boundary of the unit disk in $\mathbb{D}^{\infty}$. Take the (Euclidean) convex hull $\mathrm{Ch}(X)$ of $X$. Then the ray $R_{\beta}$ eventually enters $\mathrm{Ch}(X)$ through a crosscut $\hat{E} \subset \operatorname{Bd} \mathrm{Ch}(X)$ so that the tail of $R_{\beta}$ stays inside the shadow $\operatorname{Sh}(\hat{E})$. Observe that $\hat{E}$ is a straight segment. This defines the arc $I=(\hat{\alpha}, \hat{\gamma})$ of angles whose rays have tails in $\operatorname{Sh}(\hat{E})$, and it follows that $\beta \in I$. Consider now a hyperbolic geodesic $E_{g}$ of $\mathbb{D}^{\infty}$ (i.e., a circle arc in $\mathbb{D}^{\infty}$ connecting two points of $\mathbb{S}^{1}$ and orthogonal to $\left.\mathbb{S}^{1}\right)$ connecting $\hat{\alpha}$ and $\hat{\gamma}$, and its counterpart $E_{f}=\varphi\left(E_{g}\right)$, which is a crosscut of $X$. Clearly, $R_{\beta}$ eventually enters (and stays in) the shadow of $E_{f}$.

Lemma 4.1. There exists an essential crosscut $C^{\prime} \subset \operatorname{Sh}\left(E_{f}\right)$ with the following properties.

(1) $\operatorname{Sh}\left(C^{\prime}\right) \cap \tau(X)=\emptyset$, and hence $f\left(\operatorname{Sh}\left(C^{\prime}\right)\right) \cap X=\emptyset$.

(2) Let $\mathcal{A}\left(C^{\prime}\right)$ be the set of points in $X$ accessible from $\operatorname{Sh}\left(C^{\prime}\right)$. Then $\left.f\right|_{\left[\mathrm{Sh}\left(C^{\prime}\right) \cup \mathcal{A}\left(C^{\prime}\right)\right]}$ is one-to-one and $f\left(\operatorname{Sh}\left(C^{\prime}\right)\right)=\operatorname{Sh}\left(f\left(C^{\prime}\right)\right)$. Moreover, there exist $\varepsilon, \delta>0$ such that any defining crosscut $C_{z}, z \in R_{\beta} \cap$ $\operatorname{Sh}\left(C^{\prime}\right)$, is less than $\varepsilon$ in diameter, and any essential crosscut $C \subset$ $\mathrm{Sh}\left(C^{\prime}\right)$ less than $\varepsilon$ in diameter, maps to a crosscut $f(C)$ which is at least $\delta$-distant from $C$.

Proof. (1) Consider a defining family $C_{z}, z \in R_{\beta}$, of crosscuts of $\beta$. Then there exists a sequence of defining crosscuts $C_{z_{i}}=C_{i}, i=1,2, \ldots$, such that $z_{1}>_{\beta} z_{2}>_{\beta} \cdots$, the points $z_{i} \in R_{\beta}$ converge to $X$, and all $\bar{C}_{i}$ 's are disjoint from $\tau(X)$ (otherwise for some $z^{\prime} \in R_{\beta}$ and all $z<_{\beta} z^{\prime}$ we would have $C_{z} \cap \tau(X) \neq \emptyset$, implying that $\tau(X) \supset \Pi\left(R_{\beta}\right)=X$, a contradiction with Lemma 3.7). We may assume that $C_{1} \subset \operatorname{Sh}\left(E_{f}\right)$.

Denote by $U_{i}$ the open component of $\mathbb{C} \backslash\left[C_{i} \cup C_{i+1} \cup X\right]$ which contains points of $R_{\beta}$ located between $z_{i}$ and $z_{i+1}$. By way of contradiction (and refining the sequence $C_{i}$ if necessary) we may assume that every $U_{i}$ contains points of $\tau(X)$. Choose a point $x \in U_{i-1} \cap \tau(X)$ and a point $y \in U_{i} \cap \tau(X)$. Connect these points with an arc $A$ in $U_{i-1} \cup U_{i}$ which intersects $C_{i}$ in just one point $w$. Then choose points $s, t \in A \cap \tau(X)$ so that the subarc $B$ of $A$ with endpoints $s, t$ is disjoint from $\tau(X)$ and contains $w$.

It follows that $B$ is a crosscut of $\tau(X)$. Denote the endpoints of $C_{i}$ by $y^{\prime}, y^{\prime \prime}$. Also, denote by $W$ the shadow of $B$ in the sense of $\tau(X)$. Then it follows that one of the points $y^{\prime}, y^{\prime \prime}$ belongs to $W$ and the other not. Now, consider an internal composant $Z$ of $X$. It has points close to both $y^{\prime}$ and $y^{\prime \prime}$, hence it has points both inside $W$ and outside $W$. However, by Lemma 3.7, $\tau(X)$ is disjoint from any internal composant of $X$, a contradiction. Hence 
indeed there exists an essential crosscut $C$ such that $\operatorname{Sh}(C) \cap \tau(X)=\emptyset$, which implies that $f(\operatorname{Sh}(C)) \cap X=\emptyset$.

(2) We may assume that $C$ and all $R_{\beta}$-defining crosscuts $C_{z}, z \in R_{\beta} \cap$ $\mathrm{Sh}(C)$, are sufficiently small. By continuity and since $T(X)$ is fixed point free, the images of all these crosscuts are disjoint from the crosscuts themselves (each crosscut moves off itself by a distance which is bounded away from 0). Moreover, by (A2) and because of the properties of crosscuts, $C_{z}$ 's approach all points of $X$. Choose a crosscut $C^{\prime}$ among them so that $C^{\prime}$ is sufficiently far from $c$ and hence $f\left(C^{\prime}\right)$ is sufficiently far from $v$ so that $v \notin T\left(f\left(C^{\prime}\right)\right)$. By Lemma 3.1 then $C^{\prime}$ is a homeomorphic pullback of $f\left(C^{\prime}\right)$ and so $f\left(C^{\prime}\right)$ is a small crosscut too. Also, we can choose $C^{\prime}$ so that $c \notin \operatorname{Sh}\left(C^{\prime}\right)$.

We claim that $f\left(\operatorname{Sh}\left(C^{\prime}\right)\right)=\operatorname{Sh}\left(f\left(C^{\prime}\right)\right)$ and $\left.f\right|_{\operatorname{Sh}\left(C^{\prime}\right)}$ is a homeomorphism. Indeed, $\operatorname{Bd} f\left(\operatorname{Sh}\left(C^{\prime}\right)\right) \subset f\left(\operatorname{Bd} \operatorname{Sh}\left(C^{\prime}\right)\right)$ since $f$ is open. Hence we see that $\operatorname{Bd} f\left(\operatorname{Sh}\left(C^{\prime}\right)\right) \subset X \cup f\left(C^{\prime}\right)$. Points of $\operatorname{Sh}\left(C^{\prime}\right)$ cannot be mapped to $U_{\infty}(X)$ outside $\operatorname{Sh}\left(f\left(C^{\prime}\right)\right)$ because otherwise there will be points of $\operatorname{Bd} f\left(\operatorname{Sh}\left(C^{\prime}\right)\right)$ not in $X \cup f\left(C^{\prime}\right)$. Considering points close to $C^{\prime}$ shows that some points of $\operatorname{Sh}\left(f\left(C^{\prime}\right)\right)$ are in $f\left(\operatorname{Sh}\left(C^{\prime}\right)\right)$. Now the fact that $f(\operatorname{Sh}(C)) \cap X=\emptyset$ implies that $f\left(\operatorname{Sh}\left(C^{\prime}\right)\right) \subset \operatorname{Sh}\left(f\left(C^{\prime}\right)\right)$. Finally, if $f\left(\operatorname{Sh}\left(C^{\prime}\right)\right) \neq \operatorname{Sh}\left(f\left(C^{\prime}\right)\right)$ then there will have to be points of $\operatorname{Bd} f\left(\operatorname{Sh}\left(C^{\prime}\right)\right)$ in $\operatorname{Sh}\left(C^{\prime}\right)$, a contradiction to $\operatorname{Bd} f\left(\operatorname{Sh}\left(C^{\prime}\right)\right) \subset X \cup f\left(C^{\prime}\right)$. Thus, $f\left(\operatorname{Sh}\left(C^{\prime}\right)\right)=\operatorname{Sh}\left(f\left(C^{\prime}\right)\right)$. Hence by Lemma 3.1, $\left.f\right|_{\mathrm{Sh}\left(C^{\prime}\right)}$ is a homeomorphism.

This easily implies that $\left.f\right|_{\left[\operatorname{Sh}\left(C^{\prime}\right) \cup \mathcal{A}\left(C^{\prime}\right)\right]}$ is one-to-one too. Indeed, suppose that $z=f(x)=f(y)$ for $x \neq y \in \mathcal{A}\left(C^{\prime}\right)$. Clearly, $x \neq c, y \neq c, z \neq v$. Choose an arc $A$ joining $x$ to $y$ in $\operatorname{Sh}\left(C^{\prime}\right)$. Then $f(A)$ is a simple closed curve $S$ such that $S \cap X=\{z\}$. Choose a small arc $Q=\left[w, w^{\prime}\right] \subset S$ (in the circular order on $S$ ) so that $z \in Q, z \neq w, z \neq w^{\prime}$. Then there exist arcs $Q_{x}$ and $Q_{y}$, containing $x$ and $y$, respectively such that $f\left(Q_{x}\right)=f\left(Q_{y}\right)=Q$. Since $X$ is indecomposable, and hence contains no cutpoints, $Q_{x} \cup Q_{y} \subset$ $\operatorname{Sh}\left(C^{\prime}\right) \cup\{x\} \cup\{y\}$. This contradicts the fact that $f$ is one-to-one on $\operatorname{Sh}\left(C^{\prime}\right)$ and completes the proof.

Recall that $\varphi: \mathbb{D}^{\infty} \rightarrow U_{\infty}(X)$ is a Riemann map with $\varphi(\infty)=\infty$ (we extend $\varphi$ over the set of angles with landing rays). Next we introduce a construction from [FMOT07] simplified in our case thanks to Lemma 4.1. Take a closed round ball $B$ such that $\operatorname{int}(B) \cap X=\emptyset$ and $\operatorname{Ch}(B \cap X) \subset$ $\operatorname{Sh}\left(E_{f}\right) \cap X$ is non-degenerate. Observe that points of $B \cap X$ are accessible. Call a ball $B$ essential if $(\mathrm{Bd} B) \backslash X$ contains an essential crosscut (i.e., if $B$ "crosses over $R_{\beta}$ " from one "side" of $R_{\beta}$ to the other in an essential way).

Let $\mathcal{B}^{\prime}$ be the family of all closed round balls $B$ with $\operatorname{int}(B) \cap X=\emptyset$ and let $\mathcal{B}$ be the family of all balls $B \in \mathcal{B}^{\prime}$ such that $\operatorname{Ch}(B \cap X) \subset \operatorname{Sh}\left(E_{f}\right)$ consists of at least two points. Then $B$ is maximal in the sense of inclusion among 
all balls in $\mathcal{B}^{\prime}$. It is easy to give examples reflecting various possibilities for the sets $B \cap X$; in exceptional cases, the set $B \cap X$ could be infinite, and in truly exceptional cases it can even contain $\operatorname{arcs}$ of $\mathrm{Bd} B$. The following lemma allows us to introduce the exact shadow in the plane in which we will change the map to make a tail of $R_{\beta}$ invariant. It will be improved later and is needed here as matter of convenience to simplify the forthcoming construction.

LEMma 4.2 ([FMOT07]). There exists an essential ball $B^{*} \in \mathcal{B}$ of diameter less than $\varepsilon$ such that $\operatorname{int}\left(B^{*}\right) \subset \operatorname{Sh}\left(C^{\prime}\right)$ and $\left|B^{*} \cap X\right|=2$.

Consider the two crosscuts which are components of $\left(\mathrm{Bd} B^{*}\right) \backslash X$. Choose among them the crosscut $\widetilde{C}$ which gives rise to the shadow containing $\operatorname{int}\left(B^{*}\right)$. Clearly, $\widetilde{C}$ is essential (it suffices to consider the picture in the plane containing the set $\left.\mathbb{D}^{\infty}\right)$. Suppose that $\widetilde{C}$ has the endpoints $a, d \in X$. For the corresponding angles in $\mathbb{S}^{1}$ we use the notation $\alpha^{\prime}, \gamma^{\prime}$. Connect $\alpha^{\prime}, \gamma^{\prime}$ with a hyperbolic geodesic in $\mathbb{D}^{\infty}$ and denote this new crosscut of $\mathbb{D}^{\infty}$ by $C_{g}^{\prime}$. Denote the crosscut $\varphi\left(C_{g}^{\prime}\right)$ of $X$ by $C_{f}^{\prime}\left(C_{f}^{\prime}\right.$ replaces the crosscut $C^{\prime}$ previously introduced in Lemma 4.1 and has all the properties of $C^{\prime}$ listed in Lemma 4.1).

Now, mimicking the construction from [FMOT07] we transport the map $f$ to the set $U=\operatorname{Sh}\left(C_{g}^{\prime}\right)$ by considering a map $g(x)=\varphi^{-1} \circ f \circ \varphi(x), x \in U$. Since $\left.f\right|_{\operatorname{Sh}\left(C_{f}^{\prime}\right)}$ is a homeomorphism, so is the map $\left.g\right|_{U}$. Consider the arc of $\mathbb{S}^{1}$ defined as $\left[\alpha^{\prime}, \gamma^{\prime}\right]=\operatorname{Bd} U \cap \mathbb{D}^{\infty}$. It follows from the construction that $\beta \in\left(\alpha^{\prime}, \gamma^{\prime}\right)$.

To make a distinction, we use " $g$-" in the names of the objects in the uniformization plane (mostly these objects are $\varphi^{-1}$-images of their counterparts from the $f$-plane). Thus, the uniformization plane is called the $g$-plane; to each essential crosscut $C \subset \operatorname{Sh}\left(C_{f}^{\prime}\right)$ we associate its counterpart $\varphi^{-1}(C)$, called an essential $g$-crosscut (which is an arc connecting two points of $\mathbb{S}^{1}$, one in $\left(\alpha^{\prime}, \beta\right)$ and the other in $\left(\beta, \gamma^{\prime}\right)$, inside $U$ and intersecting $\varphi^{-1}\left(R_{\beta}\right)=R_{\beta}^{\prime}$ only once); etc. It will follow that $g\left(\varphi^{-1}(C)\right)$ is again an essential $g$-crosscut associated to the crosscut $f(C)$. The results of [FMOT07] give more information about how the map $g$ acts on $g$-crosscuts. Namely, the following theorem holds.

TheOREM 4.3 ([FMOT07, Theorems 6.5 and 9.1]). The map g can be continuously extended over the arc $\left[\alpha^{\prime}, \gamma^{\prime}\right]$. Moreover, it has the following properties:

(1) $g(\beta)=\beta$;

(2) $g$ maps the arc $\left[\alpha^{\prime}, \gamma^{\prime}\right]$ onto the arc $\left[g\left(\alpha^{\prime}\right), g\left(\beta^{\prime}\right)\right]$ homeomorphically and changes orientation (so that $g$ flips essential $g$-crosscuts contained in $\left.\operatorname{Sh}\left(C_{g}^{\prime}\right)\right)$; 
(3) for every essential crosscut $C^{\prime \prime} \subset \operatorname{Sh}\left(C_{f}^{\prime}\right)$ of diameter less than $\varepsilon$, the $g$-crosscut $g\left(\varphi^{-1}\left(C^{\prime \prime}\right)\right)$ separates $\varphi^{-1}\left(C^{\prime \prime}\right)$ from $\infty$ in $\mathbb{D}^{\infty}$.

Suppose that a closed set $Y \subset \mathbb{S}^{1}$ is chosen and consider its convex hull hypconv ${ }_{\infty}(Y)=A$ in the sense of the hyperbolic metric in $\mathbb{D}^{\infty}$. Hence hypconv $_{\infty}(Y)$ can be obtained by considering the set of components $C_{i}$ of $\mathbb{S}^{1} \backslash Y$ and joining the endpoints $a_{i}, b_{i}$ of $C_{i}$ by the geodesic in the hyperbolic metric (i.e., the intersection of the round circle through the points $a_{i}$ and $b_{i}$ with $\mathbb{D}^{\infty}$ which crosses $\mathbb{S}^{1}$ perpendicularly; see [FMOT07]). Technically, $A \cap \mathbb{D}^{\infty}$ is a subset of $\mathbb{D}^{\infty}$ which can be mapped to the $f$-plane by $\varphi$. The closure of $\varphi\left(A \cap \mathbb{D}^{\infty}\right)$ may be very complicated and not homeomorphic to $\bar{A}$. However, if the rays with the arguments from $Y$ land at distinct points of $X$ then $\bar{A}$ and $\overline{\varphi(A)}$ are homeomorphic. In this case the set $A$ will be called a $g$-cell and the set $\varphi(A)$ will be called an $f$-cell. In what follows speaking of a map $\varphi$ restricted to a cell, we always extend $\varphi$ over the boundary of the cell (by definition, $\varphi$ then remains a homeomorphism). Observe that a $g$-cell could be an arc (without endpoints) or a Jordan disk (with points on $\mathrm{Bd} \mathbb{D}^{\infty}$ removed). Moreover, if $\theta \in \mathbb{S}^{1}$ is an angle such that $R_{\theta}$ lands on $\varphi(\theta)$ then we say that $\theta$ is a degenerate $g$-cell and $\varphi(\theta)$ is a degenerate $f$-cell.

The map $\varphi$ can give a good correspondence between closed Jordan disks in the $f$-plane and in the $g$-plane. Suppose that $D$ is a closed Jordan disk in the $g$-plane such that $\varphi$ extends over the boundary of $D$. Then we call $D$ strongly homeomorphic to $\varphi(D)=A$ if $\left|D \cap \mathbb{S}^{1}\right| \geq 2$ and $\left.\varphi\right|_{D}$ is a homeomorphism. A closed Jordan disk $A$ in the $f$-plane, strongly homeomorphic to $\varphi^{-1}(A)$, is called admissible. One can transform an admissible Jordan disk $A$ in the $f$-plane to an $f$-cell $H(A)$ : choose the hyperbolic convex hull hypconv $_{\infty}\left(\overline{\varphi^{-1}(A)} \cap \mathbb{S}^{1}\right)$ of $\bar{D} \cap \mathbb{S}^{1}$ and then take its $\varphi$-image denoted $H(A)$.

A hyperbolic geodesic $\ell$ in the $g$-plane is called a g-geodesic. If $\varphi(\bar{\ell})$ is homeomorphic to $\bar{\ell}$ (i.e., to the closed interval), then $\varphi(\bar{\ell})$ is called an $f$-geodesic. Thus, boundary arcs of a $g$-cell are $g$-geodesics whose $\varphi$-images are $f$-geodesics. Clearly, two angle-arguments give rise to a $g$-geodesic whose $\varphi$-image is an $f$-geodesic if and only if the rays with these arguments land. We will also consider a degenerate geodesic with argument $\theta$, i.e. an accessible point at which the ray $R_{\theta}$ lands. Important facts concerning $f$-geodesics were established in [OT08].

Definition 4.4. Given an admissible Jordan disk $A$ in the $f$-plane, define the following sets:

(a) $P_{A}=(\operatorname{Bd} A) \cap X$;

(b) $C_{A}=A$ (if $A$ is a crosscut) or the unique component of ( $\left.\mathrm{Bd} A\right) \backslash X$ which is a crosscut with $\operatorname{int}(A) \subset \operatorname{Sh}\left(C_{A}\right)$; 
(c) $I_{A} \subset \mathbb{S}^{1}$ is an arc such that rays with arguments from $I_{A}$ have tails in $\operatorname{Sh}\left(C_{A}\right)$;

(d) $Y_{A}=\overline{\varphi^{-1}(A) \cap \mathbb{S}^{1}}$ (recall that according to the definition we have $\left.\varphi^{-1}(A) \cap \mathbb{D}^{\infty}=\operatorname{hypconv}_{\infty}\left(Y_{A}\right)\right)$.

We need to study $g$-geodesics and $f$-geodesics. As a tool we use the socalled maximal ball foliation constructed and studied in [FMOT07, OT08, KP94]. Given a crosscut $T$, denote the set $\operatorname{Sh}(T) \cup T$ by $\mathrm{Sh}^{+}(T)$. We foliate the sets $\mathrm{Sh}^{+}\left(C_{g}^{\prime}\right)$ in the $g$-plane and $\mathrm{Sh}^{+}\left(C_{f}^{\prime}\right)$ in the $f$-plane by corresponding (to each other) and specifically constructed $g$-cells and $f$-cells. However, first we need to introduce some definitions.

Definition 4.5. Suppose that there is a $g$-geodesic $T$ in the $g$-plane and that $\varphi(T)$ is an $f$-geodesic. Suppose that there is a family $\mathcal{A}$ of $g$ cells such that the following holds. For any $z \in \mathrm{Sh}^{+}(T)$ either there is a unique $g$-geodesic from the boundary of a $g$-cell $A \in \mathcal{A}$ containing $z$, or $z$ belongs to the interior of a unique $A \in \mathcal{A}$. The geodesic containing $z$ may be the intersection of two distinct $g$-cells. Then we call $\mathcal{A}$ a $(g$-)foliation $\left(\right.$ of $\left.\mathrm{Sh}^{+}(T)\right)$.

By the definition of a $g$-cell this property is transported to the $f$-plane by means of the map $\varphi$ and applies also to the family $\varphi(\mathcal{A})$ of the corresponding $f$-cells and the set $\mathrm{Sh}^{+}(\varphi(T))$. Then $\varphi(\mathcal{A})$ is called an $(f$-) foliation (of $\left.\mathrm{Sh}^{+}(\varphi(T))\right)$. The collections $\mathcal{A}, \varphi(\mathcal{A})$ are then said to be sibling foliations (of $\mathrm{Sh}^{+}(T)$ and $\mathrm{Sh}^{+}(\varphi(T))$, respectively). Also, the closure of a $g$ - or an $f$-geodesic on the boundary of a $g$ - or an $f$-cell is called a $(g$ - $)$ or $(f$ - $)$ leaf (of the corresponding foliation).

In some cases we can say much more about continuity properties of foliations. To this end we need another definition.

Definition 4.6. Suppose that there is a $g$-geodesic $T$ in the $g$-plane and that there is a foliation $\mathcal{A}$ of $\mathrm{Sh}^{+}(T)$. Then $\mathcal{A}$ is said to be uppersemicontinuous if the following holds: if a sequence of distinct $g$-cells from $\mathcal{A}$ converges, and its limit is not a point, then it converges to a $g$-leaf. If the $\varphi$-images of these $g$-cells converge to the $f$-leaf $\varphi(\ell)$, then we say that $\varphi(A)$ is upper-semicontinuous.

The sheer fact that $\mathcal{A}$ is a foliation of $\mathrm{Sh}^{+}(T)$ easily implies that $\mathcal{H}_{g}$ is upper-semicontinuous. Indeed, a sequence of $g$-cells $A_{i}$ which does not converge to a point must converge to a $g$-geodesic $\ell$ (recall that as elements of $\mathcal{A}$ the $g$-cells do not intersect inside $\mathbb{D}^{\infty}$ ). If $\ell$ is not from the boundary of an element of $\mathcal{A}$, then, since all points inside $\mathrm{Sh}^{+}(T)$ must belong to an element of $\mathcal{A}$, we will find an element of $\mathcal{A}$ which intersects $A_{i}$, a contradiction. The fact that the corresponding foliation $\varphi(\mathcal{A})$ of $\mathrm{Sh}^{+}(\varphi(T))$ is upper-semicontinuous is rather non-trivial. 
We also give a definition close to Definition 4.6 which deals with nullsequences of admissible Jordan disks. Given an admissible Jordan disk $Z$, define $\rho_{Z}(x, y)$ as the infimum of diameters of open $\operatorname{arcs} J(x, y)=J$ in $U_{\infty}$ whose closures $\bar{J}$ connect $x, y$ and are homotopic to $\operatorname{arcs} I(x, y)=I \subset Z$ connecting $x, y$ inside $Z \cap U_{\infty}$ under a homotopy in $U_{\infty}$ fixing $x$ and $y$ (the definition is inspired by the Mazurkiewicz metric discussed later).

LEMMA 4.7. There exists a universal constant $K$ with the following property. Suppose that $A$ is an admissible Jordan disk in the $f$-plane such that for any two points $x, y$ of $\bar{A} \cap X=P_{A}$ we have $\rho_{A}(x, y) \leq M$. Then $\operatorname{diam}(H(A)) \leq K M$.

Proof. Take points $x, y \in P_{A}$. By the assumptions of the lemma and by Pommerenke [Pom92, Theorem 4.2], there exists a constant $K^{\prime}$ such that the diameter of the $f$-geodesic $G(x, y)$ is at most $K^{\prime} M$. The geodesic $G(x, y)$ is chosen to be homotopic to one of the $\operatorname{arcs} I(x, y)=I$ connecting $x, y$ inside $Z \cap U_{\infty}$ under a homotopy in $U_{\infty}$ fixing $x$ and $y$. Now, given two points $u, v \in H(A)$, connect them by the $f$-geodesic $I$ and then extend it to the $f$-geodesic $I_{1}$ which connects points $u \in \operatorname{Bd} H(A)$ and $v \in \operatorname{Bd} H(A)$. Choose the $f$-geodesics $I_{2}, I_{3}$ from the boundary of $H(A)$ such that $u \in I_{2}$ and $v \in I_{3}$. Fix an endpoint of $I_{2}$, an endpoint of $I_{3}$, and the $f$-geodesic $I_{4}$ which connects them. Then by the triangle inequality and by the above, $|I| \leq\left|I_{1}\right| \leq\left|I_{2}\right|+\left|I_{3}\right|+\left|I_{4}\right| \leq 3 K^{\prime} M$. So, any two points of $H(A)$ can be connected by an $f$-geodesic of diameter at most $3 K^{\prime} M$, which completes the proof.

In what follows by a null-sequence we mean a sequence of sets whose diameters converge to zero.

Definition 4.8. Suppose that $\mathcal{A}$ and $\varphi(\mathcal{A})$ are sibling foliations. Suppose that given a sequence of $g$-cells $A_{i} \in \mathcal{A}$ and the corresponding sequence $\varphi\left(A_{i}\right)$ of $f$-cells, $\left\{A_{i}\right\}$ is a null-sequence if and only if $\left\{\varphi\left(A_{i}\right)\right\}$ is a null-sequence. Then we call the foliations $\mathcal{A}, \varphi(\mathcal{A})$ null preserving.

We can finally single out pairs of foliations we want to work with.

Definition 4.9. Suppose that there is a crosscut $T$ in the $g$-plane and that $\varphi(T)$ is a crosscut of $X$. Moreover, suppose that $\mathcal{A}, \varphi(A)$ are sibling foliations of $\mathrm{Sh}^{+}(T)$ and $\mathrm{Sh}^{+}(\varphi(T))$ respectively which are uppersemicontinuous and null preserving. Then we say that they form a canonical pair (of foliations) or just that they are canonical.

Observe that elements of foliations are always $g$-cells and $f$-cells. If $\mathcal{A}, \varphi(\mathcal{A})$ are canonical then for distinct $g$-cells $A^{\prime}, A^{\prime \prime} \in \mathcal{A}$ only the following cases are possible: (1) $A^{\prime}, A^{\prime \prime}$ have a common $g$-geodesic in their boundaries but are otherwise disjoint: (2) the closures of $A^{\prime}, A^{\prime \prime}$ have a unique point of 
$\mathbb{S}^{1}$ in common; (3) the closures of $A^{\prime}, A^{\prime \prime}$ are disjoint. This implies that the sets $\overline{A^{\prime}} \cap \mathbb{S}^{1}, \overline{A^{\prime \prime}} \cap \mathbb{S}^{1}$ are unlinked, i.e. one of them is contained in a component of the complement to the other (except for the endpoints). Observe that the collection of geodesics in the boundaries of all the $g$-cells $A \in \mathcal{A}$ is a lamination of $\operatorname{Sh}(T)$ (cf. [Thu85]).

In general $\varphi$ is far from being continuous near $\mathbb{S}^{1}$. However, the existence of canonical foliations allows us to use a version of continuity of $\varphi$ justified by Lemma 4.10 below. By $d(a, b)$ we understand the standard Euclidian distance between two points $a, b \in \mathbb{C}$.

Lemma 4.10. Let $\mathcal{A}, \varphi(\mathcal{A})$ be canonical foliations. Then the family of restrictions $\left\{\left.\varphi\right|_{\bar{A}}\right\}_{A \in \mathcal{A}}$ is equicontinuous: for any $\varepsilon^{\prime}$ there exists $\delta^{\prime}$ such that if $x^{\prime}, z^{\prime} \in \bar{A}, A \in \mathcal{A}$ and $d\left(x^{\prime}, z^{\prime}\right) \leq \delta^{\prime}$ then $d\left(\varphi\left(x^{\prime}\right), \varphi\left(z^{\prime}\right)\right) \leq \varepsilon^{\prime}$.

Proof. By way of contradiction suppose that $x_{i}^{\prime}, z_{i}^{\prime} \in \overline{A_{i}}, A_{i} \in \mathcal{A}$, are two sequences with $d\left(x_{i}^{\prime}, z_{i}^{\prime}\right) \rightarrow 0$ (here $A_{i} \in \mathcal{A}$ is a sequence of $g$-cells from $\mathcal{A}$ ), but $d\left(\varphi\left(x_{i}^{\prime}\right), \varphi\left(z_{i}^{\prime}\right)\right) \nrightarrow 0$. Refining the sequences, we may assume that $\lim x_{i}^{\prime}=\lim z_{i}^{\prime}=a$ while $\varphi\left(x_{i}^{\prime}\right) \rightarrow x_{f}, \varphi\left(z_{i}^{\prime}\right) \rightarrow z_{f}$ and $x_{f} \neq z_{f}$. Let us show that $A_{i}$ is not a null-sequence. Indeed, if $A_{i}$ is a null-sequence, then $\varphi\left(A_{i}\right)$ is a null-sequence (since $\mathcal{A}, \varphi(A)$ are canonical) and $d\left(\varphi\left(x_{i}^{\prime}\right), \varphi\left(z_{i}^{\prime}\right)\right) \rightarrow 0$, a contradiction. Since the foliations are canonical we may assume that $A_{i}$ converge to a $g$-geodesic $\ell$ on the boundary of a $g$-cell. Then $\varphi\left(A_{i}\right)$ converge to the $f$-geodesic $\varphi(\ell)$, and hence the fact that $\lim x_{i}^{\prime}=\lim z_{i}^{\prime}=a$ implies that $\lim \varphi\left(x_{i}^{\prime}\right)=\lim \varphi\left(z_{i}^{\prime}\right)$, a contradiction with $x_{f}^{\prime} \neq z_{f}^{\prime}$.

An important example of a canonical pair of foliations comes from the construction of the family $\mathcal{B}$ of maximal closed, round balls introduced right before Lemma 4.2 (the corresponding tools can be found in [FMOT07] and [KP94]). Let $\mathcal{H}_{g}$ be the family of all sets of the form hypconv ${ }_{\infty}\left(\varphi^{-1}(B \cap X)\right)$ $\subset \operatorname{Sh}\left(C_{g}^{\prime}\right), B \in \mathcal{B}$. Denote by $\mathcal{H}_{f}$ the corresponding family of all sets $H(B) \subset \operatorname{Sh}\left(C_{f}^{\prime}\right), B \in \mathcal{B}$.

Theorem 4.11 ([FMOT07], see also [KP94]). The foliations $\mathcal{H}_{g}$ and $\mathcal{H}_{f}$ are sibling foliations of $\mathrm{Sh}^{+}\left(C_{g}^{\prime}\right)$ and $\mathrm{Sh}^{+}\left(C_{f}^{\prime}\right)$ respectively.

The main next step is to show that $\mathcal{H}_{g}, \mathcal{H}_{f}$ form a canonical pair of foliations. The following corollary follows from [FMOT07].

COROLlary 4.12. Suppose that there is a sequence $A_{n}$ of distinct g-cells from $\mathcal{H}_{g}$. Then the following facts hold.

(1) If $A_{n}$ converge to a hyperbolic geodesic $\ell=\alpha \beta$, then $\ell$ is a g-leaf of $\mathcal{H}_{g}, \varphi(\ell)$ is an $f$-leaf of $\mathcal{H}_{f}$, and $\overline{\varphi\left(A_{n}\right)}$ converge to $\overline{\varphi(\ell)}$.

(2) If $A_{n}$ is a null-sequence, then $\varphi\left(A_{n}\right)$ is a null-sequence.

Proof. Each of the $f$-cells $\varphi\left(A_{n}\right)$ corresponds to a maximal ball $B_{n} \in \mathcal{B}$. In case (1) the balls $B_{n}$ have diameters bounded away from zero and may 
be assumed to converge to a non-degenerate maximal ball $B$. In case (2), by way of contradiction and after refining the sequence, we may assume that $A_{n}$ converge to a point $\alpha=\beta \in \mathbb{S}^{1}$ while $\varphi\left(A_{n}\right)$ converge to a nondegenerate continuum. By Lemma 4.7 the fact that the diameters of $\varphi\left(A_{n}\right)$ are bounded away from zero implies that the diameters of $B_{n} \cap \varphi\left(A_{n}\right)$ are bounded away from zero (otherwise by Lemma 4.7 the diameters of $\varphi\left(A_{n}\right)$ go to zero). Hence again the balls $B_{n}$ converge to a maximal ball $B \in \mathcal{B}$. By Lemma 4.2 in [FMOT07], $\varphi\left(A_{n}\right) \subset B_{n}$. By Lemma 5.1 in [FMOT07], $\lim \varphi\left(A_{n}\right) \cap X=\{\alpha, \beta\}$. Hence $\lim \overline{\varphi\left(A_{n}\right)}=T \subset B$. So far the situation has been similar for both cases, but now we consider them separately.

(1) From geometric considerations in the $f$-plane, $T \subset \varphi(\ell) \cup \operatorname{Imp}(\alpha) \cup$ $\operatorname{Imp}(\beta)$. The fact that $\varphi\left(A_{n}\right) \cap X$ converges to $\{\varphi(\alpha), \varphi(\beta)\}$ and $\operatorname{int}\left(B_{n}\right)$ does not contain $\varphi(\alpha), \varphi(\beta)$ implies that there is an arc $J_{n} \subset \operatorname{Bd} B$ such that $J_{n} \subset \operatorname{int}\left(B_{n}\right)$ (thus, $\left.J \cap X=\emptyset\right)$ and $\lim J_{n}=J$ is an arc in $\operatorname{Bd} B$ from $\alpha$ to $\beta$. Hence $J \cap X=\{\varphi(\alpha), \varphi(\beta)\}$. Let $K=(\operatorname{Bd} B) \backslash J$ and suppose that $K$ contains a point $x \in X \backslash\{\varphi(\alpha), \varphi(\beta)\}$. Let $C_{n}$ be the component of $B_{n} \backslash \overline{\varphi(\ell)}$ which contains $\varphi\left(A_{n}\right)$ (recall that $\varphi(\alpha)$ and $\varphi(\beta)$ are outside the interior of $\left.B_{n}\right)$. Then it is easy to observe that there exists $\varepsilon>0$ such that $d\left(x, C_{n}\right)>\varepsilon$. Hence $\lim \varphi\left(A_{n}\right)=\overline{\varphi(\ell)}$.

(2) By the above and by the conditions of the lemma, $T \subset B \cap X$. Since $T$ is a continuum, it follows that $T \subset \mathrm{Bd} B$ is a non-degenerate arc. Clearly, this contradicts the fact that $A_{n}$ is a null-sequence.

In Theorem 4.13 we summarize the above results.

TheOREM 4.13 ([FMOT07], see also [KP94]). The sibling foliations $\mathcal{H}_{g}$ and $\mathcal{H}_{f}$ form a canonical pair.

Proof. By the remark after Definition 4.6, $\mathcal{H}_{g}$ is upper-semicontinuous. Suppose that $\varphi\left(A_{n}\right)$ is a convergent sequence of distinct $f$-cells whose limit is not a point. By Corollary 4.12(2) the diameters of $A_{n}$ are bounded away from zero. Hence we may assume that $A_{n}$ converge to a $g$-leaf of $\mathcal{H}_{g}$. Now by Corollary 4.12(1), $\varphi\left(A_{n}\right)$ converge to an $f$-leaf of $\mathcal{H}_{f}$ and $\mathcal{H}_{f}$ is uppersemicontinuous. On the other hand, if $\varphi\left(A_{n}\right)$ is a null-sequence of $f$-cells in $\mathcal{H}_{f}$ while $A_{n}$ is not a null-sequence of $g$-cells, then, after refining $A_{n}$ and by Corollary 4.12(1), we may assume that $A_{n}$ converge to a $g$-leaf $\ell$ and $\varphi\left(A_{n}\right)$ converge to its non-degenerate image $\varphi(\ell)$, a contradiction.

Let us state a useful corollary of results of [FMOT07].

Corollary 4.14. Let $\xi \in\left(\alpha^{\prime}, \gamma^{\prime}\right) \subset \mathbb{S}^{1}$ be an angle which is not an endpoint of a g-leaf of $\mathcal{H}_{g}$. Then there exists a sequence $B_{i}$ of $R_{\xi}$-essential balls from $\mathcal{B}$ with radii converging to zero and such that $\operatorname{Sh}\left(C_{f}^{\prime}\right) \supset \operatorname{Sh}\left(C_{H\left(B_{1}\right)}\right) \supset$ $\operatorname{Sh}\left(C_{H\left(B_{2}\right)}\right) \supset \cdots$ and $\operatorname{hypconv}_{\infty}\left(\varphi^{-1}\left(B_{i} \cap X\right)\right) \rightarrow\{\xi\}$. 
By the above, the foliations $\mathcal{H}_{g}$ and $\mathcal{H}_{f}$, induced by maximal balls, form a canonical pair of foliations. The next lemma allows us to extend this result to a more general situation. However, first we need to define the Mazurkiewicz metric $\rho$. Consider the set $G(X)=G$ of all pairs $(\varphi(\alpha), \alpha)$ such that the ray $R_{\alpha}$ lands on $\varphi(\alpha)$. Given $t=(\varphi(\alpha), \alpha)$ and $w=(\varphi(\beta), \beta)$, define $\rho(t, w)$ as follows. If $t=w$ set $\rho(t, w)=0$. If $t \neq w$, let $\rho(t, w)$ be the infimum of the diameters of open $\operatorname{arcs} J$ in $U_{\infty}$ whose closures are closed $\operatorname{arcs}$ from $\varphi(\alpha)$ to $\varphi(\beta)$ or simple closed curves containing $\varphi(\alpha)=\varphi(\beta)$ such that $\varphi^{-1}(J)$ is homotopic to the geodesic $\alpha \beta \in \mathbb{D}^{\infty}$ under a homotopy fixing $\alpha, \beta$.

Lemma 4.15. Let $T$ be a g-geodesic and $\varphi(T)$ be an $f$-geodesic. Let $\mathcal{A}, \varphi(\mathcal{A})$ be sibling foliations of $\mathrm{Sh}^{+}(T), \mathrm{Sh}^{+}(\varphi(T))$ respectively. Consider a sequence $A_{i}$ of distinct $g$-cells of $\mathcal{A}$ which converge to a (degenerate) $g$-leaf $\ell$ such that $\overline{\varphi\left(A_{i}\right)} \cap X \rightarrow \overline{\varphi(\ell)} \cap X$ in the Mazurkiewicz metric. Then the closed $f$-cells $\overline{\varphi\left(A_{i}\right)}$ converge to the closed (degenerate) f-leaf $\overline{\varphi(\ell)}$.

Proof. Consider first the case when $\ell=\theta$ is a degenerate $g$-geodesic. By the conditions of the lemma it follows that Lemma 4.7 applies to $\varphi\left(A_{i}\right)$. Thus, the diameters of $\varphi\left(A_{i}\right)$ converge to zero and $\varphi\left(A_{i}\right)$ converge to $\varphi(\theta)$ as required.

Suppose now that $\ell=\alpha \beta$ and $\alpha \neq \beta$. Clearly, $\varphi(\ell) \subset \lim \sup \varphi\left(A_{i}\right)$. Partition the sequence $A_{i}$ into two sequences which converge to $\ell$ from opposite sides (in $\mathbb{D}^{\infty}$ ) and consider them separately. Without loss of generality assume that there are $\alpha_{i}, \beta_{i} \in \overline{A_{i}} \cap \mathbb{S}^{1}$ such that $\alpha_{i}<\alpha<\beta$ $<\beta_{i}$ and there are no points of $A_{i} \cap \mathbb{S}^{1}$ between $\alpha_{i}$ and $\alpha$ and between $\beta$ and $\beta_{i}$. Let $\alpha_{i} \alpha$ be the $g$-geodesic connecting $\alpha_{i}$ and $\alpha$. Define the left wing $\operatorname{Imp}^{-}(\alpha)=\bigcap \overline{\operatorname{Sh}\left(\varphi\left(\alpha \alpha_{i}\right)\right)}$ of the impression $\operatorname{Imp}(\alpha)$; define the right wing $\operatorname{Imp}^{+}(\beta)$ of the impression $\operatorname{Imp}(\beta)$ similarly. Then it is easy to see that $\lim \sup \varphi\left(A_{i}\right) \subset \varphi(\ell) \cup \operatorname{Imp}^{-}(\alpha) \cup \operatorname{Imp}^{+}(\beta)$.

It follows from the conditions of the lemma and Theorem 4.2 from [Pom92] that $\varphi\left(\alpha_{i} \alpha\right) \rightarrow \varphi(\alpha)$ and $\varphi\left(\beta_{i} \beta\right) \rightarrow \varphi(\beta)$. Choose $\varepsilon>0$. Choose $i$ such that for any $n \geq i$ we have the following: (1) $\operatorname{diam}\left(\varphi\left(\alpha_{i} \alpha\right)\right)+$ $\operatorname{diam}\left(\varphi\left(\beta \beta_{i}\right)\right)<\varepsilon$, and (2) if we denote the set $\varphi\left(\alpha_{i}, \alpha\right) \cap A_{n}$ by $A_{n}^{\alpha}$, then the $\rho$-distance between the set $\varphi\left(A_{n}^{\alpha}\right)$ and $\varphi(\alpha)$ is less than $\varepsilon$, and similarly for $\beta$ and the similarly defined sets $A_{n}^{\beta}$. The set $A_{n} \backslash\left[\alpha \alpha_{i} \cup \beta \beta_{i}\right]$ consists of three components. Let $T_{n}$ be the one of them containing $A_{n}^{\alpha}, L_{n}$ be the one of them containing $A_{n}^{\beta}$, and $M_{n}$ be the remaining third component. Then $\lim M_{n}=\ell$ and $\lim \varphi\left(M_{n}\right)=\varphi(\ell)$.

Consider the hyperbolic convex hull $Q_{n}^{\alpha}$ of the points of $A_{n}^{\alpha}, \alpha_{i}, \alpha$. Then $T_{n} \subset Q_{n}^{\alpha}$. By the choice of $i$ any two points of $\varphi\left(Q_{n}^{\alpha}\right) \cap X$ can be joined by an arc in $U_{\infty}$ of diameter less than $2 \varepsilon$. Then by Lemma 4.7, $\operatorname{diam}\left(\varphi\left(T_{n}\right)\right) \leq$ $\operatorname{diam}\left(\varphi\left(Q_{n}^{\alpha}\right)\right) \leq 2 K \varepsilon$. Hence $\lim \varphi\left(T_{n}\right)=\{\varphi(\alpha)\}$. Similarly, $\lim \varphi\left(L_{n}\right)=$ $\{\varphi(\beta)\}$. Thus, $\lim \varphi\left(A_{n}\right)=\varphi(\ell)$ as desired. 
Observe that Lemma 4.15 gives another proof of Corollary 4.12. Indeed, let $A_{n}$ be a sequence of distinct $g$-cells of $\mathcal{H}_{g}$ taken from Corollary 4.12. As in the initial part of the proof of that corollary, we may assume that $A_{n}$ converge to a $g$-leaf $\alpha \beta$ and the corresponding maximal balls $B_{n}$, associated with $f$-cells $\varphi\left(A_{n}\right)$, have radii bounded away from zero and converge to a non-degenerate ball $B$. Then it follows from geometric considerations that the $\rho$-distance between two "clusters" of $\varphi\left(A_{n}\right) \cap B_{n}=\varphi\left(A_{n}\right) \cap X$ and the corresponding points $\alpha$ or $\beta$ goes to zero. By Lemma 4.15, this implies that $\overline{\varphi\left(A_{n}\right)} \rightarrow \overline{\varphi(\alpha \beta)}$ as desired.

Denote by $\mathcal{G}$ the family of all $g$-leaves in $\mathcal{H}_{g}$; denote the union of all such leaves by $\mathcal{G}^{*}$. Note that if $B \in \mathcal{B}$ and $\left|P_{B}\right|=|B \cap X|=2$, then $\operatorname{hypconv}_{\infty}\left(\varphi^{-1}(B \cap X)\right) \in \mathcal{G}$ and hypconv $\log _{\infty}\left(\varphi^{-1}(B \cap X)\right)$ is a $g$-leaf. A set hypconv $_{\infty}(B)$ which is not a $g$-leaf is said to be a $g$-gap. Thus, if $\left|P_{B}\right|=$ $|B \cap X| \geq 3$, then hypconv ${ }_{\infty}\left(\varphi^{-1}(B \cap X)\right)$ is a $g$-gap and all geodesics in its boundary are $g$-leaves. The $\varphi$-images of $g$-leaves are called $f$-leaves. Their entire family is denoted by $\mathcal{G}_{f}$. The $\varphi$-images of $g$-gaps are called $f$-gaps. The $\varphi$-image of $\mathcal{G}^{*}$ is denoted by $\mathcal{G}_{f}^{*}$.

We will show in Theorem 4.16 that we can modify the map $f$ to a branched covering map $f^{*}$ such that $\left.f^{*}\right|_{R_{\beta} \cap \operatorname{Sh}(C)}: R_{\beta} \cap \operatorname{Sh}(C) \rightarrow R_{\beta}$ is an embedding for some $R_{\beta}$-essential crosscut $C$.

THEOREM 4.16. There exists an $R_{\beta}$-essential crosscut $C$ and a branched covering map $f^{*}: \mathbb{C} \rightarrow \mathbb{C}$ of degree -2 such that $\left.f^{*}\right|_{\mathbb{C} \backslash \operatorname{Sh}(C)}=\left.f\right|_{\mathbb{C} \backslash \operatorname{Sh}(C)}$, $\left.f^{*}\right|_{\operatorname{Sh}(C)}: \operatorname{Sh}(C) \rightarrow f(\operatorname{Sh}(C))$ is a homeomorphism with $f^{*}\left(R_{\beta} \cap \operatorname{Sh}(C)\right)=$ $R_{\beta} \cap \operatorname{Sh}(f(C))$, and there exists $x_{0} \in R_{\beta}$ such that $f^{*}(x)>_{\beta} x$ for all $x<_{\beta} x_{0}$.

Proof. The first step in the proof is to refine the sequence $B_{i}$ from Corollary 4.14 so that it has the following property. Consider $H\left(B_{1}\right)$ and the crosscut $C_{H\left(B_{1}\right)}$, "farthest away from $X$ " among crosscuts from $\operatorname{Bd} H\left(B_{1}\right)$. Let $I_{H\left(B_{1}\right)}=I_{B_{1}}=(\alpha, \gamma) \subset \mathbb{S}^{1}$. By Corollary 4.14 , we may choose $B_{1}$ so that the following holds. Denote the endpoints of $C_{H\left(B_{1}\right)}$ by $p_{1}$ and $q_{1}$. Choose $B_{1}$ so that both $C_{H\left(B_{1}\right)}$ and $f\left(C_{H\left(B_{1}\right)}\right)$ are contained in $\operatorname{Sh}\left(C_{f}^{\prime}\right)$. In addition choose it so that the $f$-geodesic joining $f\left(p_{1}\right), f\left(q_{1}\right)$ is also contained in $\operatorname{Sh}\left(C_{f}^{\prime}\right)$.

Let $Q=\operatorname{Sh}\left(C_{H\left(B_{1}\right)}\right)$. It will also be useful to consider $\varphi^{-1}(Q)$. Clearly, $\operatorname{Bd} \varphi^{-1}(Q)$ is the union of $\varphi^{-1}\left(C_{H\left(B_{1}\right)}\right)$ and the arc $I_{B_{1}}=(\alpha, \gamma) \subset \mathbb{S}^{1}$. By Theorem 4.13 the restrictions of the foliations $\mathcal{H}_{g}$ and $\mathcal{H}_{f}$ to $\varphi^{-1}(Q)$ and $Q$ form a canonical pair. For simplicity, we still denote them $\mathcal{H}_{g}$ and $\mathcal{H}_{f}$.

The idea is to modify the map $g$ so that a tail of the radial ray $\varphi^{-1}\left(R_{\beta}\right)$ $=r_{\beta}$ is fixed and then transport it back to the $f$-plane. The new map $g^{*}$ will coincide with $g$ on $\mathbb{S}^{1}$. The modification of $g$ takes place inside $\operatorname{Sh}\left(C_{g}^{\prime}\right)$ while 
the corresponding modification of $f$ takes place in $\operatorname{Sh}\left(C_{f}^{\prime}\right)$. We construct $g^{*}$ in a few steps. First, we construct a foliation whose elements are the future images (under the map $g^{*}$ when it is defined) of the associated elements of $\mathcal{H}_{g}$. Then $g^{*}$ is defined inside elements of $\mathcal{H}_{g}$ so that it satisfies the standard continuity and extension conditions and keeps a tail of $R_{\beta}$ invariant as desired.

Elements of the new foliation are associated to elements of $\mathcal{H}_{g}$ as follows. Observe that by Theorem 4.3 the map $g$ on $(\alpha, \gamma) \subset \mathbb{S}^{1}$ is a homeomorphism with a fixed repelling point $\beta$; the map $g$ flips points around $\beta$. Therefore the fact that all sets $\bar{A} \cap \mathbb{S}^{1}, A \in \mathcal{H}_{g}$, are unlinked implies that the sets $g\left(\bar{A} \cap \mathbb{S}^{1}\right)$, $A \in \mathcal{H}_{g}$, are unlinked too. Denote the convex hull of the set $g\left(\bar{A} \cap \mathbb{S}^{1}\right)$ in the hyperbolic metric in $\mathbb{D}^{\infty}$ by $Z_{A}$. We conclude that the sets $Z_{A}$ form a foliation $\mathcal{H}_{g}^{*}$ of the set $\operatorname{Sh}(K)$ where $K$ is the $g$-geodesic in $\mathbb{D}^{\infty}$ connecting points $g(\alpha), g(\gamma)$.

For angles $u, v \in \mathbb{S}^{1}$ let $u v$ be the $g$-geodesic connecting $u$ and $v$. We want to define a homeomorphic extension $g^{*}$ from $\operatorname{Sh}(\alpha \gamma)$ to $\operatorname{Sh}(g(\alpha) g(\gamma))$. We do this so that, for each $g$-cell $A, g^{*}$ maps $A \in \mathcal{H}_{g}$ onto $Z_{A}$ as an orientation preserving homeomorphism and coincides with $g$ on $\bar{A} \cap \mathbb{S}^{1}$. In particular, a boundary $g$-geodesic of $A$ maps to the corresponding boundary $g$-geodesic of $Z_{A}$ so that the endpoints are mapped as the map $g$ prescribes. We can then extend this map over all gaps by mapping the barycenter of each gap to the barycenter of the image gap and subsequently "coning" the map on the gap (see [OT08]).

Let $A \cap r_{\beta} \neq \emptyset$. By Theorem 4.3 then $Z_{A}$ intersects $r_{\beta}$ farther away from $\mathbb{S}^{1}$ than $A$ in the sense of the order on $r_{\beta}$. It is easy to see that then $g^{*}$ can be designed so that in addition to the above we have $g^{*}\left(r_{\beta} \cap A\right)=$ $r_{\beta} \cap Z_{A}$. By Theorem 4.13 the entire tail of $r_{\beta}$ inside $\varphi^{-1}(Q)$ is covered by the sets $A \cap r_{\beta}, A \in \mathcal{H}_{g}$, hence the new map $g^{*}$ maps $\varphi^{-1}(Q)$ onto $\operatorname{Sh}(K)$ so that $g^{*}\left(r_{\beta} \cap \varphi^{-1}(Q)\right)=r_{\beta} \cap \operatorname{Sh}(K)$. Since by Theorem 4.3 the map $g$ is continuous, the newly constructed map $g^{*}$ can be constructed to be continuous on $\overline{\varphi^{-1}(Q)}$. Clearly, $g^{*}$ is a homeomorphism. The next claim is crucial for the proof of the theorem.

Claim 1. The foliations $\mathcal{H}_{g}^{*}, \varphi\left(\mathcal{H}_{g}^{*}\right)$ of $\operatorname{Sh}(K)$ are canonical.

Proof of Claim 1. Let $A$ be a $g$-cell. Let us show that $\varphi\left(\overline{Z_{A}}\right)$ and $\overline{Z_{A}}$ are homeomorphic. Indeed, by the above $\left.g^{*}\right|_{A}$ is a homeomorphism from $A$ to $Z(A)$. Since $\mathcal{H}_{g}$ and $\mathcal{H}_{f}$ are a canonical pair, $A$ and $\varphi(A)$ are strongly homeomorphic. By Lemma 4.1, $f(\overline{\varphi(A)})$ and $\varphi(\bar{A})$ are homeomorphic. This implies that $\varphi\left(\overline{Z_{A}}\right)$ and $\overline{Z_{A}}$ are homeomorphic to each other and to $\bar{A}$ and $\overline{\varphi(A)}$.

Hence $\mathcal{H}_{g}^{*}, \varphi\left(\mathcal{H}_{g}^{*}\right)$ are sibling foliations. Moreover, by the remark right after Definition $4.6, \mathcal{H}_{g}^{*}$ is upper-semicontinuous. We need to show that the foliation $\varphi\left(\mathcal{H}_{g}^{*}\right)$ is upper-semicontinuous. 
Indeed, consider a sequence of $g$-cells $A_{i} \in \mathcal{H}_{g}^{*}$ which converges to a $g$-geodesic $\ell$ in the boundary of a $g$-cell $A \in \mathcal{H}_{g}^{*}$. Denote the endpoints ("endangles") of $\ell$ by $\theta^{\prime}$ and $\theta^{\prime \prime}$. As in the remark right after Lemma 4.15, the geometric considerations imply that the $\rho$-distance between two "clusters" of the set $\varphi\left(A_{n}\right) \cap X$ and the appropriate points $\varphi\left(\theta^{\prime}\right), \varphi\left(\theta^{\prime \prime}\right)$ goes to zero. It follows from Lemma 4.15 that then the $f$-cells $\varphi\left(\overline{A_{i}}\right)$ converge to the $f$-geodesic in the boundary of $\varphi(\bar{A})$ connecting the landing points of the rays $R_{\theta^{\prime}}$ and $R_{\theta^{\prime \prime}}$.

It remains to show that under $\varphi$ null-sequences of cells in $\mathcal{H}_{g}^{*}$ and in $\varphi\left(\mathcal{H}_{g}^{*}\right)=\mathcal{H}_{f}^{*}$ correspond to each other. One way it immediately follows: if a sequence of $f$-cells is null but their $\varphi$-preimages form a sequence which is not null, then we can refine the latter to get a sequence converging to a nontrivial set inside $\mathbb{D}^{\infty}$. Its $\varphi$-image has to be contained in the limit of the just introduced $f$-cells which can only be a point, a contradiction. Now, suppose that $A_{i} \in \mathcal{H}_{g}^{*}$ is a null sequence. We may assume that $\overline{A_{i}} \cap \mathbb{S}^{1}=g\left(\overline{D_{i}} \cap \mathbb{S}^{1}\right)$ where $D_{i} \in \mathcal{H}_{g}$. Since $g$ is a homeomorphism, $D_{i}$ form a null sequence. Then by Theorem 4.13 , the $f$-cells $\varphi\left(D_{i}\right)$ form a null-sequence too. Therefore by continuity and Lemma 4.1, $\operatorname{diam}_{a}\left(f\left(\varphi\left(D_{i}\right)\right)\right) \rightarrow 0$. By Lemma 4.7, and by the construction, then $\operatorname{diam}_{a}\left(\varphi\left(A_{i}\right)\right) \rightarrow 0$ as desired. Hence $\mathcal{H}_{g}^{*}$ is canonical and Claim 1 is proven.

We define $g^{*}$ so that it maps $A \in \mathcal{H}_{g}$ onto $Z_{A}$ as an orientation preserving homeomorphism and coincides with $g$ on $\bar{A} \cap \mathbb{S}^{1}$. In particular, a boundary $g$-geodesic of $A$ maps to the corresponding boundary $g$-geodesic of $Z_{A}$ so that the endpoints are mapped as the map $g$ prescribes. We can then extend this map over all gaps by mapping the barycenter of each gap to the barycenter of the image gap and subsequently coning the map on the gap. Suppose that $A \cap r_{\beta} \neq \emptyset$. It follows from Theorem 4.3 that then $Z_{A}$ intersects $r_{\beta}$ farther away from $\mathbb{S}^{1}$ than $A$ in the sense of the order on $r_{\beta}$. It is easy to see that then $g^{*}$ can be designed so that in addition to the above we have $g^{*}\left(r_{\beta} \cap A\right)=r_{\beta} \cap Z_{A}$. By Theorem 4.13 the entire tail of $r_{\beta}$ inside $\varphi^{-1}(Q)$ is covered by the sets $A \cap r_{\beta}, A \in \mathcal{H}_{g}$, hence the new map $g^{*}$ maps $\varphi^{-1}(Q)$ onto $\operatorname{Sh}(K)$ so that $g^{*}\left(r_{\beta} \cap \varphi^{-1}(Q)\right)=r_{\beta} \cap \operatorname{Sh}(K)$. Since by Theorem 4.3 the map $g$ is continuous, the newly constructed map $g^{*}$ can be constructed to be continuous on $\overline{\varphi^{-1}(Q)}$.

Now the map $g^{*}$ can be transported to the $f$-plane by means of the map $\varphi$. To begin with, the new map $f^{*}$ is defined only on $Q$ as follows: $f^{*}=\varphi \circ g^{*} \circ \varphi^{-1}$. Moreover, by the construction the map $f^{*}$ is also defined on entire sets $\bar{A}, A \in \mathcal{H}_{f}$. Still, there are two problems which need to be resolved before we complete the proof of the theorem.

First, we need to extend $f^{*}$ from $Q$, beyond the crosscut $C_{H\left(B_{1}\right)}$ which serves as the gates into the shadow $Q$, onto the strip between $C_{H\left(B_{1}\right)}$ and $C_{f}^{\prime}$. 
To see that this is possible, notice that under $f^{*}$ the $f$-geodesic crosscut $C_{H\left(B_{1}\right)}$ is mapped so that (a) $f^{*}\left(C_{H\left(B_{1}\right)}\right)=\varphi(K)$ is an $f$-geodesic crosscut in whose shadow $C_{H\left(B_{1}\right)}$ is contained, and (b) $f$ and $f^{*}$ on $C_{H\left(B_{1}\right)}$ are homotopic outside $T(X)$. Clearly, the map $f^{*}$ can be extended to the region between $C_{H\left(B_{1}\right)}$ and $C_{f}^{\prime}$ as a homeomorphism so that its action coincides with that of $f$ on $\mathbb{C} \backslash \operatorname{Sh}\left(C_{f}^{\prime}\right)$ and with $f^{*}$ on $C_{H\left(B_{1}\right)}$.

Second, we define $f^{*}$ on the entire $\mathbb{C}$ as $f^{*}$ (already defined above on $\left.\operatorname{Sh}\left(C_{f}^{\prime}\right)\right)$ and $f$ elsewhere. We need to show that the map $f^{*}$ is continuous. This needs to be proven only at points of $X$. Indeed, let $x_{i} \rightarrow x, x \in X$, and show that then $f^{*}\left(x_{i}\right) \rightarrow f(x)$. We may assume that $x_{i} \notin T(X)$. Then for each $i$ we can choose an $f$-cell $L_{i} \in \mathcal{H}_{f}$ with $x_{i} \in L_{i}$. To each $L_{i}$ we associate the corresponding $g$-cell $M_{i}=\varphi^{-1}\left(L_{i}\right)$, which by the construction is the convex hull of the set $\overline{M_{i}} \cap \mathbb{S}^{1}$ in the hyperbolic metric on $\mathbb{D}^{\infty}$.

Suppose that $\operatorname{diam}\left(L_{i}\right) \rightarrow 0$. Then the sets $\varphi^{-1}\left(L_{i}\right)$ form a null-sequence (since $\mathcal{H}_{g}, \mathcal{H}_{f}$ are canonical), hence their $g^{*}$-images form a null-sequence of $g$-cells from $\mathcal{H}_{g}^{*}$, hence by Claim 1 the sets $\varphi\left(g^{*}\left(\varphi^{-1}\left(L_{i}\right)\right)\right)=f^{*}\left(L_{i}\right)$ form a null-sequence of $f$-cells from $\mathcal{H}_{f}^{*}$. Since $x_{i} \rightarrow x$ and $L_{i}$ form a nullsequence, we can find points $z_{i} \in \overline{L_{i}} \cap X$ with $z_{i} \rightarrow x$. Then since $f$ does not change on $X, f^{*}\left(z_{i}\right)=f\left(z_{i}\right) \rightarrow f(x)$. On the other hand, $d\left(f^{*}\left(x_{i}\right), f^{*}\left(z_{i}\right)\right)$ $\rightarrow 0$ because $\left\{f^{*}\left(L_{i}\right)\right\}$ is a null-sequence. Hence $f^{*}\left(x_{i}\right) \rightarrow f^{*}(x)=f(x)$ as desired.

Suppose now that $f^{*}\left(x_{i}\right) \nrightarrow f^{*}(x)=f(x)$. We may assume (by the previous paragraph) that diam $L_{i} \geq \varepsilon^{\prime \prime}$ for some $\varepsilon^{\prime \prime}>0$. Then since $\mathcal{H}_{f}$ and the corresponding foliation $\mathcal{H}_{g}$ are canonical, we may assume that $L_{i} \rightarrow$ $L \in \mathcal{H}_{f}$ and $M_{i} \rightarrow M \in \mathcal{H}_{g}$ with both $L, M$ being non-degenerate. Clearly, the points $\varphi^{-1}\left(x_{i}\right)$ converge to a point $\varphi^{-1}(x) \in \bar{M} \cap \mathbb{S}^{1}$, which implies that there are points $z_{i} \in \overline{L_{i}} \cap X$ such that $z_{i} \rightarrow x$ and $\varphi^{-1}\left(z_{i}\right) \rightarrow \varphi^{-1}(x)$. Therefore, by the construction, $d\left(g^{*}\left(x_{i}\right), g^{*}\left(z_{i}\right)\right) \rightarrow 0$. By Lemma 4.10 this implies that $d\left(\varphi\left(g^{*}\left(x_{i}\right)\right), \varphi\left(g^{*}\left(z_{i}\right)\right)\right)=d\left(f^{*}\left(x_{i}\right), f^{*}\left(z_{i}\right)\right) \rightarrow 0$. Since $f^{*}\left(z_{i}\right)=$ $f\left(z_{i}\right) \rightarrow f^{*}(x)=f(x)$, we finally conclude that $f^{*}\left(x_{i}\right) \rightarrow f^{*}(x)$ as desired. Thus, the map $f^{*}$ has all the required properties and the theorem is proven.

5. Converging arcs and fixed points. By (A1)-(A7), $X$ is an indecomposable continuum containing no subcontinua $Y$ with $f(Y) \subset T(Y)$. In particular, $X$ contains no invariant subcontinua not equal to $X$. By the construction, if we prove our Main Theorem for $f^{*}$, it will hold for $f$ too. Thus, in what follows we denote the map $f^{*}$ constructed in Section 4 by simply $f$. Also, set $R_{\beta}=R$. We deal a lot with subsegments of $R$ and from now on skip the subscript $\beta$ in denoting them (so that $[a, b]$ means in fact $[a, b]_{\beta}$ etc.). Similarly we denote the order $<_{\beta}$ in $R$ simply by $<$ (the situation considered in this section allows us to do so without causing any ambiguity). 
Sometimes, however, we need to deal with subarcs of other arcs/rays/lines, not contained in $R$. In that case we indicate this with a subscript; thus, if $T$ is an arc/ray/line and $u, v \in T$ then by $[u, v]_{T}$ we mean the closed subarc of $T$ with endpoints $u, v$ (for rays $R_{\alpha}$ we use the usual notation $[a, b]_{\alpha}$ ). Denoting subsets of $R$ we use $\infty$ in the obvious sense (thus, $(x, \infty)$ is the subray of $R$ consisting of all points $y \in R$ with $y>x$ ).

By Section 4, we may assume that for some $z \in R$ the tail $(0, z]$ of $R$ is invariant in the sense that $\left.f\right|_{(0, z]}:(0, z] \rightarrow(0, f(z)]$ is an embedding such that for all $x \in(0, z], f(z)>z$. The ray $R$ is ordered from infinity towards $X$; if $u, v \in R$ and $u<v$, say that $v$ is $R$-closer to $X$ ( $u$ is closer to $\infty$ ) than $u$. We also say $R$-closer speaking of points on $R$ and meaning the order on $R$.

We need the following lemma.

Lemma 5.1. If $Z \subset X$ is nowhere dense in $X$ then for any $n$ the set $Z \cup f(Z) \cup \cdots \cup f^{n}(Z)$ is nowhere dense in $X$ too. Hence, since $X \cap \tau(X)$ is a closed and nowhere dense subset of $X$, for any $n \in \mathbb{Z}^{+}$there exists an open set $U \subset X$ such that for each $i=0,1, \ldots, n, f^{i}(U) \cap \tau(X)=\emptyset$.

Proof. Given a closed ball $B$ not containing the critical image, we have $f^{-1}(B)=B^{\prime} \cup B^{\prime \prime}$ with both $B^{\prime}, B^{\prime \prime}$ homeomorphic to $B$. Since $Z \cap B^{\prime}$ is nowhere dense in $B^{\prime} \cap X$, then $f\left(Z \cap B^{\prime}\right)$ is nowhere dense in $f\left(B^{\prime} \cap X\right) \subset$ $B \cap X$. Similarly, $f\left(Z \cap B^{\prime \prime}\right)$ is nowhere dense in $B \cap X$ too. Hence $f(Z) \cap B$, which is the union of two sets nowhere dense in $B \cap X$, is nowhere dense in $B \cap X$. This implies that $f(Z)$ is nowhere dense in $X$ and proves, inductively, the first claim of the lemma.

Set $Z=X \cap \tau(X)$. Then the complement to $Z \cup f(Z) \cup \cdots \cup f^{n}(Z)$ is a dense open subset $W$ of $X$ (by the first paragraph). On the other hand, $W$ consists of the points $x$ such that the sets $\{x\}, f^{-1}(x), \ldots, f^{-n}(x)$ are disjoint from $Z$. Hence any point $y \in f^{-n}(x)$ is such that $y, f(y), \ldots$, $f^{n}(y)=x$ do not belong to $Z$. Hence if we take a small neighborhood $U$ of $y$ it will satisfy the requirements of the lemma.

Now we are ready to prove our main theorem.

TheOREM 5.2. Suppose that $f: \mathbb{C} \rightarrow \mathbb{C}$ is a branched covering map such that the absolute value of the degree is at most 2 , and let $Y$ be a continuum such that $f(Y) \subset T(Y)$. Then one of the following holds.

(1) The map $f$ has a fixed point in $T(Y)$.

(2) The continuum $Y$ contains a fully invariant indecomposable continuum $X$ such that $X$ contains no subcontinuum $Z$ with $f(Z) \subset Z$; moreover, in this case degree $(f)=-2$.

Proof. As before, we assume that (1) does not hold while the standard assumptions (A1)-(A7) apply to an indecomposable continuum $X \subset Y$. We 
also assume that the map has already been modified according to Theorem 4.16 and that therefore there exists a ray $R=R_{\beta}$ with all properties of $R_{\beta}$ as well as properties listed in Theorem 4.16. We may assume that $X$ is a non-degenerate continuum containing no invariant subcontinua and such that $\left.f\right|_{T(X)}$ is fixed point free. Note that by (A7), $f^{-1}(X)=X \cup \tau(X) \supsetneq X$ is a continuum.

By Lemma 3.7, the set $X \cap \tau(X)$ is nowhere dense in $X$ while $X \backslash \tau(X)=$ $Q$ is a dense open subset of $X$. By Lemma 5.1 we can choose a point $p \in Q$ so that $f(p)=q \in Q, f(q) \in Q, f^{2}(q) \in Q$. We may assume that $p$ is not equal to $c$ and its first preimages. Thus we can choose a small neighborhood $V$ of $p$ such that $\left.f^{3}\right|_{V}$ is a homeomorphism. Set $U=f(V), U^{\prime}=f(U)$, $U^{\prime \prime}=f^{2}(U)$; we may assume that $X \cap\left(V \cup U \cup U^{\prime} \cup U^{\prime \prime}\right) \subset X \backslash \tau(X)$. Since the principal set of $R$ is $X$, the sibling ray $\tau(R)$ is dense in $\tau(X)$ in the sense that $\overline{\tau(R)} \backslash \tau(R)=\tau(X)$. This implies that for some $\delta>0$ the sibling ray $\tau(R)$ does not come closer than $\delta$ to $p, q, f(q)$ or $f^{2}(q)$. Hence we may assume that $V, U, U^{\prime}$ and $U^{\prime \prime}$ are all disjoint from $\tau(R)$.

Choose an $R$-defining family of crosscuts $C_{t}$ (see Section 3 ). Since $R$ converges to $X$, there is a point $r \in V \cap R$ with $\overline{C_{r}} \subset U$. Choose $r \in R$ to satisfy a few conditions. First, we may assume that $(r, \infty)$ is a vertical line and $C_{r}$ is a horizontal segment. By Theorem 4.16, we may assume that $f^{4}(r) \in R$ and $\left.f\right|_{\operatorname{Sh}\left(C_{f^{3}(r)}\right)}: \operatorname{Sh}\left(C_{f^{3}(r)}\right) \rightarrow \operatorname{Sh}\left(f\left(\operatorname{Sh}\left(C_{f^{3}(r)}\right)\right.\right.$ is a homeomorphism so that $f$ maps points on $R \cap \operatorname{Sh}\left(C_{f^{3}(r)}\right)$ to points on $R$ closer to $\infty$. Let $W=\operatorname{Sh}\left(C_{f^{3}(r)}\right)$. We may also assume that $W$ contains no fixed points of $f$.

In the forthcoming arguments we move along the ray $R$ towards $X$ and use the terms like "after", "before" etc. in the appropriate sense. Figure 1 may help the reader to visualize the following construction. Extend $C_{r}$ a bit to the left while removing the part located to the right of $r$ to create an arc $G \subset V$ disjoint from $(r, \infty)$. We may assume that $(0, r)$ intersects $G$ infinitely often. To see this, take a sequence of points on $R$ converging to the endpoint of $G$ distinct from $r$, draw an arc through them all which ends at the left endpoint of $G$ and is disjoint from $C_{r}$, and then add this arc to $G$. The added arc can be chosen arbitrarily small. Then shorten $G$ a little by choosing its endpoint $t$ distinct from $r$ as the first point after $r$ (closest to $r$ in the sense of the order on $R$ ) of $(0, r)$ intersecting the just extended $G$. Then $[r, t] \cup G$ is a Jordan curve, and we may assume that $X$ meets both the unbounded component and the bounded component of $\mathbb{C} \backslash\{[r, t] \cup G\}$. Indeed, we can choose a point $y \in R$ and an essential crosscut $C_{y}$ such that $\overline{C_{y}} \cap \overline{C_{r}}=\emptyset$. Then we can construct $G$ so that $G \backslash C_{r}$ is very small and hence is disjoint from $C_{y}$. This implies that one of the two endpoints of $C_{y}$ is inside the unbounded component and the other is in the bounded component of $\mathbb{C} \backslash\{[r, t] \cup G\}$. 


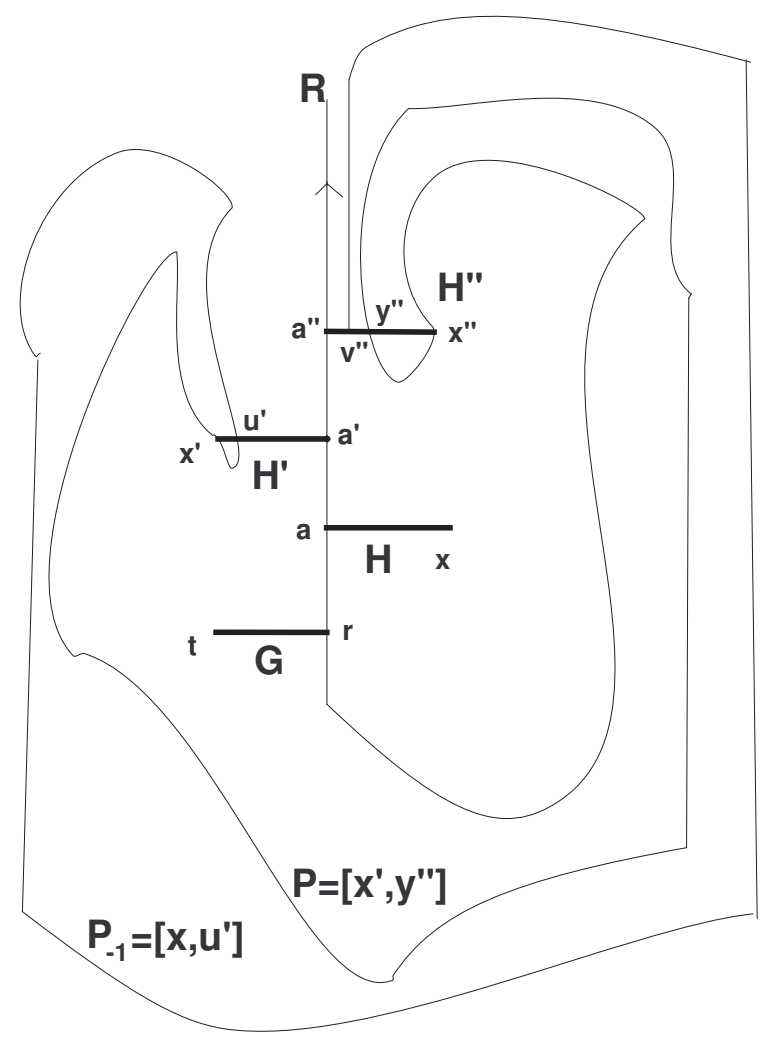

Fig. 1. The ray $R=R_{\beta}$

By Section 4 the image of $G$ is the arc $f(G)=H \subset U$ which grows out of $R$ at $f(r)=a$ and sticks out of $R$ to the right (the other endpoint of $H$ is $f(t)=x)$. By Section 4 the image of $H$ is the arc $f(H)=H^{\prime} \subset U^{\prime}$ which grows out of $R$ at $f(a)=a^{\prime}$ and sticks out of $R$ to the left (the other endpoint of $H^{\prime}$ is $\left.f(x)=x^{\prime}\right)$. Finally, we consider the $\operatorname{arc} f^{2}(H)=H^{\prime \prime} \subset U^{\prime \prime}$ which grows out of $R$ at $f^{2}(a)=a^{\prime \prime}$, sticks out of $R$ to the right and has the other endpoint $f^{2}(x)=x^{\prime \prime}$. By the choice of $U$ the segments $H, H^{\prime}$ and $H^{\prime \prime}$ are pairwise disjoint. Moreover, by the choice of $q$ the points of $X \cap\left(G \cup H \cup H^{\prime} \cup H^{\prime \prime}\right)$ have no siblings in $X$. To simplify the language we may assume that $G, H, H^{\prime}, H^{\prime \prime}$ are all horizontal segments.

By the choice of $G$ the set $G \cap R$ is infinite. Hence the sets $H \cap R, H^{\prime} \cap R$ and $H^{\prime \prime} \cap R$ are infinite. Also, recall that by the above choices $\tau(R)$ is disjoint from $V, U, U^{\prime}, U^{\prime \prime}$ and hence from $H$ and $H^{\prime}$. Clearly, there are irreducible subsegments of $R$ connecting points of $H$ and $H^{\prime}$. We call such segments of $R$ prime segments, or simply primes. Two distinct primes intersect at most in one of their endpoints (in this case one can call them concatenated). 
The idea of what follows is to consider primes and their pullbacks. We show that there is a "monotone" sequence of primes $P_{0}, P_{-1}, \ldots$ in the sense that their endpoints on $H$ and $H^{\prime}$ are ordered monotonically. Moreover, the primes have the property that $f\left(P_{-n-1}\right) \subset P_{-n}$. Then these primes converge to a limit continuum $K$ with $f(K) \subset K$. However, the monotonicity implies that $P_{0}$ cuts the limit continuum $K$ off some points of $X$ and hence $K \neq X$, a contradiction with the assumption that $X$ contains no proper invariant subcontinuum. Defining the desired sequence of primes requires some purely geometric considerations in the plane.

Our arguments are based upon the observation that if moving along $R$ from infinity towards $X$ we meet a point, then before that we must have met the image of that point; this is based upon the fact that the points in $R$ map towards infinity (i.e., $f(z)>z$ for $z \in R \cap W$ ). By the construction $R$ passes through $x$. Let us show that this is the first time $R$ intersects $H$ after $r$. Indeed, otherwise there is a point $z>x, z \in H \cap R$. The point $z$ has the preimage $z^{\prime} \in G$ which cannot belong to $\tau(R)$ because $\tau(R)$ is disjoint from $U$. Hence $z^{\prime} \in R$. Moreover, $z^{\prime}>t$ because $z>x$. This contradicts the choice of $t$. Similarly, $x^{\prime}$ and $x^{\prime \prime}$ are the first times after $r$ when the ray $R$ intersects the $\operatorname{arcs} H^{\prime}$ and $H^{\prime \prime}$ respectively. This in turn implies, by the same argument, that in fact $x^{\prime}$ is the first point at which $R$ hits $f^{-1}\left(H^{\prime \prime}\right)$.

Clearly, the arc $\left[a^{\prime \prime}, x^{\prime \prime}\right] \subset R$ together with $H^{\prime \prime}$ forms a Jordan curve $S$ which encloses an open Jordan disk $D$. Moreover, since both $H$ and $H^{\prime \prime}$ are located to the right of $R$ and since $H$ is disjoint from $S$ except for $a$ we see that $H \backslash\{a\} \subset D$. Now, the ray $R$ may have other points of intersection with $H^{\prime \prime}$ after $x^{\prime \prime}$ and before it hits $H^{\prime}$ for the first time at $x^{\prime}$. Denote by $y^{\prime \prime}$ the last point of $H^{\prime \prime}$ before $x^{\prime}$. Clearly, the ray points up as it finally exits $D$ at $y^{\prime \prime}$ for otherwise the point $y^{\prime \prime}$ would not be the last point on $R$ before it hits $H^{\prime}$ (we use the fact that $R$ can only exit $D$ through $H^{\prime \prime}$ and that $H^{\prime}$ is outside $D)$.

Let $P$ be the subarc of $R$ given by $\left[y^{\prime \prime}, x^{\prime}\right]$. It follows that $\left[a^{\prime \prime}, y^{\prime \prime}\right]_{H^{\prime \prime}} \cup P \cup$ $H^{\prime} \cup\left[a^{\prime}, a^{\prime \prime}\right]$ is a simple closed curve $T$ which encloses a disk $\widehat{D}$ (recall that intervals without subscripts are subarcs of $R$ ). Observe that the simple closed curves $S=\left[a^{\prime \prime}, x^{\prime \prime}\right] \cup H^{\prime \prime}$ and $T$ have an arc $\left[a^{\prime}, a^{\prime \prime}\right] \cup\left[a^{\prime \prime}, y^{\prime \prime}\right]_{H^{\prime \prime}}$ in common and therefore form a $\theta$-curve. Observe also that by the construction, $D \subset \widehat{D}$ (see Figure 1).

Repeating the above arguments with obvious changes in notation we see that after $x^{\prime}$ the ray $R$ may have intersections with $H^{\prime}$, then it finally goes off $H^{\prime}$ at a point $u^{\prime} \in R \cap H^{\prime}$ and then it hits $H$ for the first time at the point $x$. We claim that as $R$ goes off $H^{\prime}$ at $u^{\prime}$, it points up and moves outside $\widehat{D}$. Indeed, suppose otherwise. Then the ray $R$ after $u^{\prime}$ goes inside $\widehat{D}$ and crosses $H$ at $x$ for the first time while not crossing $H^{\prime}$ before that. Consider 
the Jordan disk $D^{\prime}$ whose boundary is formed by $H^{\prime}$ and the subsegment $\left[a^{\prime}, x^{\prime}\right]$ of $R$. Since $R$ cannot intersect itself and, by the assumption, it does not intersect $H^{\prime}$ after $u^{\prime}$ before it intersects $H$, we see that $\left[u^{\prime}, x\right] \subset D^{\prime}$. However by the construction the point $x$ lies outside $D^{\prime}$, a contradiction.

Thus, after $u^{\prime}$ the ray $R$ goes up. By the choice of $u^{\prime}$ as the last point on $H^{\prime}$ on $R$ before $R$ hits $H$, it follows that $R$ has to penetrate $D \ni x$ through $H^{\prime \prime}$ in order to reach out to $x$. Since $P=\left[y^{\prime \prime}, x^{\prime}\right]$ shields the subarc $\left[y^{\prime \prime}, x^{\prime \prime}\right]_{H^{\prime \prime}}$ of $H^{\prime \prime}$ from $R$, the first point of intersection between $R$ and $H^{\prime \prime}$ after $u^{\prime}$ has to be a point $v^{\prime \prime} \in\left[a^{\prime \prime}, y^{\prime \prime}\right]_{H^{\prime \prime}}$. Clearly, $R$ approaches $H^{\prime \prime}$ from above before it hits $H^{\prime \prime}$ at $v^{\prime \prime}$. As we continue towards $H$, the ray $R$ after $v^{\prime \prime}$ may have more intersections with $H^{\prime \prime}$, but then it finally hits $H$ at $x$. This creates our first prime $P_{0}=\left[u^{\prime}, x\right]$ on which we have a subarc $\left[u^{\prime}, v^{\prime \prime}\right]$ with $v^{\prime \prime} \in\left[a^{\prime \prime}, y^{\prime \prime}\right]_{H^{\prime \prime}}$. The prime $P_{0}$, together with the arc $\left[u^{\prime}, a^{\prime}\right]_{H^{\prime}} \cup\left[a^{\prime}, a\right] \cup H$, forms a simple closed curve $Y$ which encloses a disk $L_{0}$.

Let us now define the first pullback of $P_{0}$. It follows that the "zigzag" arc $I=H^{\prime} \cup\left[a^{\prime}, a\right] \cup H$ can be pulled back to the arc $J=G \cup[a, r] \cup H$ (this pullback is simply a restriction of the corresponding branch of the inverse function which is a homeomorphism). Observe that all points of $R \cap I$ then pull back to points of $R \cap J$ (otherwise there will have to be points of $\tau(R)$ in $J$, which is impossible). In particular, there exists a point $u \in H \cap R$ with $f(u)=u^{\prime}$. This pullback can then be extended onto $P_{0}$, say, starting at $x$ and then by continuity. Let us show that this results in a subarc of $R$ which connects $t \in G$ to $u \in H$. Indeed, under this pullback the point $x$ pulls back to $t$. Recall that by the construction $R$ hits $G$ at $t$ for the first time. Hence $P_{0}$ pulls back to an $\operatorname{arc} Q$ which at least around $t$ is a subarc of $R$, and hence overall (as a set) is a subarc of $R$ too. The other endpoint of this subarc of $R$ should be the unique preimage of $u^{\prime}$ belonging to $R$, and by the shown above this can only be the point $u$. Moreover, since at $u^{\prime}$ the prime $P_{0}$ points up, so does the $\operatorname{arc} Q$ at $u$.

Observe also that $Q$ cannot intersect $H$ at more than one point since otherwise its image $P_{0}$ will intersect $H^{\prime}$ at more than one point. Therefore the $\operatorname{arc} Q$ exits $L_{0}$ at $u$ only to penetrate back into $L_{0}$ later through $H^{\prime}$ in order to reach out to $t \in G$. Denote by $s$ the point of $H^{\prime}$ closest to $u$ on $Q$ and show that $s=v^{\prime}$ is the unique preimage of $v^{\prime \prime}$ on $H^{\prime}$. Indeed, since $\tau(R)$ is "far away" from $V, U, U^{\prime}$ and $U^{\prime \prime}$, then $\tau\left(G \cup H \cup H^{\prime} \cup H^{\prime \prime}\right)$ is disjoint from $R$. In particular, there are no points of $\tau\left(H^{\prime}\right)$ in $[u, s) \subset Q$. On the other hand, there are no points of $H^{\prime}$ in $[u, s)$ by the choice of $s$. Therefore there are no points of $f^{-1}\left(H^{\prime \prime}\right)$ in $[u, s)$, which implies that there are no points of $H^{\prime \prime}$ in $\left[u^{\prime}, f(s)\right)$ while $f(s) \in H^{\prime \prime}$. By the definition of $v^{\prime \prime}$ this implies that $f(s)=v^{\prime \prime}$ and hence $s=v^{\prime}$ as desired. Moreover, $u \in H$ is closer to $a$ on $H$ than $x$ and $v^{\prime}$ is closer to $a^{\prime}$ on $H^{\prime}$ than $u^{\prime}$. Indeed, the 
former is obvious. Also, as we pointed out before, $Q$ exits $L_{0}$ at $u$ and then it can only come back into $L_{0}$ through $\left[u^{\prime}, a^{\prime}\right]_{H^{\prime}}$ so that indeed $v^{\prime}$ is closer to $a^{\prime}$ on $H^{\prime}$ than $u^{\prime}$.

The arc $\left[u, v^{\prime}\right] \subset Q$ is then declared to be the next prime $P_{-1}$. By the construction, its image is a subarc of $P_{0}$. Moreover, $P_{-1}$ connects $H$ and $H^{\prime}$ in a specific way, namely so that the initial small segments at the endpoints of $P_{-1}$ point up with respect to the horizontal $\operatorname{arcs} H$ and $H^{\prime}$ respectively. To make the notation consistent let us from now on denote the endpoints of $P_{0}$ by $\alpha_{0}=x, \beta_{0}=u^{\prime}$ and the endpoints of $P_{-1}$ by $\alpha_{1}=u$ and $\beta_{1}=v^{\prime}$. Observe that $G \subset L_{0}$. The endpoints of $P_{-1}$ are located so that $\alpha_{1}$ is closer to $a$ on $H$ than $\alpha_{0}$, and $\beta_{1}$ is closer to $a^{\prime}$ on $H^{\prime}$ than $\beta_{0}$.

The above established properties of primes can be used in the inductive process showing that we can construct a sequence of primes with similar properties. Namely, suppose that we already have a finite sequence of pairwise disjoint primes $P_{0}, P_{1}, \ldots, P_{-n}$ such that the following holds.

(1) $P_{-i}=\left[\beta_{i}, \alpha_{i}\right]$ with $\beta_{i} \in H^{\prime}, \alpha_{i} \in H$ and $P_{-i} \cap\left(H^{\prime} \cup H\right)=\left\{\alpha_{i}, \beta_{i}\right\}$;

(2) for each $i, 0 \leq i \leq n-1$, the point $\beta_{i+1}$ is closer to $a^{\prime}$ than the point $\beta_{i}$ on the $\operatorname{arc} H^{\prime}$

(3) for each $i, 0 \leq i \leq n-1$, the point $\alpha_{i+1}$ is closer to $a$ than the point $\alpha_{i}$ on the $\operatorname{arc} H$;

(4) for each $i, 0 \leq i \leq n-1$, we have $f\left(\alpha_{i+1}\right)=\beta_{i}$;

(5) the initial segments of $P_{-i}$ at the endpoints of $P_{-i}$ point up;

(6) for each $i, 0 \leq i \leq n-1$, we have $f\left(P_{-(i+1)}\right) \subset P_{-i}$.

Let us show that then we can construct the next prime $P_{-n-1}$ so that all these properties are satisfied. First, though, we locate a few points using the fact that $\left.f^{3}\right|_{V}$ is a homeomorphism. Since $f\left(\alpha_{n}\right)=\beta_{n-1}$, we see that $f\left(\left[a, \alpha_{n}\right]_{H}\right)=\left[a^{\prime}, \beta_{n-1}\right]_{H^{\prime}}$. Hence there is a preimage of $\beta_{n} \in H^{\prime}$ in $H$ between $a$ and $\alpha_{n}$. Denote this preimage $\alpha_{n+1}$. Also, choose $\zeta_{n+1}$ on $G$ so that $f\left(\zeta_{n+1}\right)=\alpha_{n}$. Finally, set $\left[\alpha_{n}, a\right]_{H} \cup\left[a, a^{\prime}\right] \cup\left[a^{\prime}, \beta_{n}\right]_{H^{\prime}}=Q_{n}$. Then it follows from the location of the primes that $P_{-n} \cup Q_{n}=E_{n}$ is a Jordan curve which encloses a Jordan disk $L_{n}$, and $L_{0} \subset L_{1} \subset \cdots \subset L_{n}$. Moreover, $G \subset L_{0}$.

The point $\beta_{n}$ has two preimages, $\alpha_{n+1}$ and $\tau\left(\alpha_{n+1}\right)$. One of them belongs to $R$, the other to $\tau(R)$. Since $\tau(R)$ is disjoint from $V, U, U^{\prime}, U^{\prime \prime}$ we have $\alpha_{n+1} \in R$. Similarly we see that $\zeta_{n+1} \in R$. Hence the pullback $S_{n}$ of $P_{-n}$ within $R$ (we can talk about it because by Theorem 4.16 we assume that a tail of $R$ is invariant) connects $\zeta_{n+1}$ and $\alpha_{n+1}$. Moreover, $S_{n}$ points up at the points $\zeta_{n+1}$ and $\alpha_{n+1}$ because so does $P_{n}$ at their images, i.e. at the points $\alpha_{n}$ and $\beta_{n}$.

It follows that at $\alpha_{n+1}$ the $\operatorname{arc} S_{n}$ exits $L_{n}$ and that $S_{n}$ intersects $H$ only at $\alpha_{n+1}$ (otherwise $P_{n}$ would intersect $H^{\prime}$ at more than one point $\beta_{n}$ ). Since 
the other endpoint of $S_{n}$ is $\zeta_{n+1} \in L_{n}$, it must enter back into $L_{n}$, and by the above it can only do so through $H^{\prime}$ closer to $a^{\prime}$ than $\beta_{n}$ (the rest of $H^{\prime}$ is shielded from $S_{n}$ by $P_{-n}$ ). Follow $S_{n}$ from $\alpha_{n+1}$ on towards $\zeta_{n+1}$ until it meets $H^{\prime}$ for the first time. Denote by $\beta_{n+1}$ the point of $S_{n}$ which belongs to $H^{\prime}$ and is closest to $\alpha_{n+1}$ on $\mathbb{R}$. Then the arc $\left[\alpha_{n+1}, \beta_{n+1}\right]=P_{-n-1}$ satisfies all the conditions on primes listed above. Thus, we have been able to make the induction step which proves the existence of an infinite sequence of primes $\left\{P_{-i}\right\}_{i=0}^{\infty}$ with the above properties.

By the construction the sequence of primes $\left\{P_{-i}\right\}$ converges to a continuum which we denote $Z$. Indeed, the endpoints of the primes $\alpha_{n}, \beta_{n}$ converge to points $\alpha \in H \cap X, \beta \in H^{\prime} \cap X$ respectively. Choose $Z$ to be the limit of a subsequence of primes, then choose a small neighborhood $M$ of $Z$, and then choose $P_{-N}$ so that the $\operatorname{arc}\left[\alpha, \alpha_{N}\right]_{H} \cup P_{-N} \cup\left[\beta_{n}, \beta\right]_{H^{\prime}} \subset M$. It follows that then the Hausdorff distance between $P_{-k}$ and $Z$ for any $k>N$ must be small, which implies that $Z$ is the limit (in the Hausdorff metric) of the sequence of primes $P_{-n}$.

Obviously, $Z \subset X$. Moreover, by the construction there are points of $X$ inside $L_{0}$ while $Z$ is disjoint from $L_{0}$. Therefore $Z \neq X$. However, by continuity the fact that $f\left(P_{-(i+1)}\right) \subset P_{-i}$ for every $i$ implies that $f(Z) \subset Z$, which contradicts the minimality of $X$. Hence we may finally conclude that the assumption of $X$ not being fully invariant fails. In other words, $X$ is fully invariant (i.e. $f^{-1}(X)=X=f(X)$ ) as desired.

We would like to make a few concluding remarks here. The fact that $X$ is fully invariant allows us to work with the entire uniformization plane. Recall that $\varphi: \mathbb{D}^{\infty} \rightarrow U_{\infty}(X)$ is a Riemann map with $\varphi(\infty)=\infty$. Then the map $f$ is transported to the uniformization plane on which we obtain a well-defined map $g(x)=\varphi^{-1} \circ f \circ \varphi(x), x \in \mathbb{D}^{\infty}$. This construction is exactly the same as the standard construction from complex dynamics; it was used in a more complicated situation of a non-fully invariant continuum in [FMOT07] as well as above in Section 4 (though in that case the map $g$ was not considered on the entire $\mathbb{D}^{\infty}$ ).

By the results of [FMOT07], $f$ induces a covering map $G: \mathbb{S}^{1} \rightarrow \mathbb{S}^{1}$ on the circle of prime ends of $T(X)$ (i.e., $g$ continuously extends over $\mathbb{S}^{1}=\operatorname{Bd} \mathbb{D}^{\infty}$ as a covering map of the circle). It is easy to check that $\operatorname{deg}(G)=-2$. Hence, $G$ has exactly three fixed points $\left\{\alpha_{1}, \alpha_{1}, \alpha_{3}\right\}$ in $\mathbb{S}^{1}$. Suppose that $C_{n}$ is a fundamental chain of crosscuts of the prime end $\alpha_{j}$. Since diam $\left(C_{n}\right) \rightarrow 0$ and $f$ is fixed point free on $T(X)$, for all $n$ sufficiently large, $f\left(C_{n}\right) \cap C_{n}=\emptyset$. Hence from that point on either $f\left(C_{n}\right)$ separates $C_{n}$ from infinity in $\mathbb{C} \backslash T(X)$ (the points are "repelled" from $X$ in the sense of the order on the ray $R_{\alpha_{j}}$, in which case we have the so-called outchannel defined more precisely in [FMOT07]), or $C_{n}$ separates $f\left(C_{n}\right)$ from infinity in $\mathbb{C} \backslash T(X)$ (the points are 
"attracted" towards $X$ in the sense of the order on the ray $R_{\alpha_{j}}$, in which case we have the so-called inchannel defined more precisely in [FMOT07]). By [FMOT07] there exists exactly one outchannel, therefore two of the fixed prime ends must correspond to inchannels. Hence the induced map $G$ on the circle of prime ends has degree -2 , exactly one repelling fixed point and two attracting fixed points. This details the dynamics in the neighborhood of $X$.

Acknowledgements. We would like to thank the referee for useful remarks.

The first author was partially supported by NSF grant DMS-0456748. The second author was partially supported by NSF grant DMS-0405774.

\section{References}

[Aki99] V. Akis, On the plane fixed point problem, Topology Proc. 24 (1999), 15-31.

[BO06] A. Blokh and L. Oversteegen, Monotone images of Cremer Julia sets, Houston J. Math., to appear; arXiv:0809.1193.

[Bel67] H. Bell, On fixed point properties of plane continua, Trans. Amer. Math. Soc. 128 (1967), 539-548.

[Bel78] -, A fixed point theorem for plane homeomorphisms, Fund. Math. 100 (1978), 119-128; see also: Bull. Amer. Math. Soc. 82 (1976), 778-780.

[Bon04] M. Bonino, A Brouwer like theorem for orientation reversing homeomorphisms of the sphere, Fund. Math. 182 (2004), 1-40.

[Bro12] L. E. J. Brouwer, Beweis des ebenen Translationssatzes, Math. Ann. 72 (1912), 35-41.

[Bro84] M. Brown, A new proof of Brouwer's lemma on translation arcs, Houston J. Math. 10 (1984), 35-41.

[CL51] M. L. Cartwright and J. E. Littlewood, Some fixed point theorems, Ann. of Math. 54 (1951), 1-37.

[CMT] D. Childers, J. Mayer, M. Tuncali and E. Tymchatyn, Indecomposable continua and the Julia sets of rational maps, in: Contemp. Math. 396, Amer. Math. Soc., 2006, 1-20.

[Fat87] A. Fathi, An orbit closing proof of Brouwer's lemma on translation arcs, Enseign. Math. 33 (1987), 315-322.

[FMOT07] R. J. Fokkink, J. C. Mayer, L. G. Oversteegen, and E. D. Tymchatyn, The plane fixed point problem, arXiv:0805.1184v2.

[Fra92] J. Franks, A new proof of the Brouwer plane translation theorem, Ergodic Theory Dynam. Systems 12 (1992), 217-226.

[Gui94] L. Guillou, Théorème de translation plane de Brouwer et généralisations du théorème de Poincaré-Birkhoff, Topology 33 (1994), 331-351.

[Ili70] S. D. Iliadis, Location of continua on a plane and fixed points, Vestnik Moskov. Univ. Mat. 25 (1970), no. 4, 66-70 (in Russian).

[Kra74] J. Krasinkiewicz, On internal composants of indecomposable plane continua, Fund. Math. 84 (1974), 255-263.

[KP94] R. S. Kulkarni and U. Pinkall, A canonical metric for Möbius structures and its applications, Math. Z. 216 (1994), 89-129.

[Mil00] J. Milnor, Dynamics in One Complex Variable, 2nd ed., Vieweg, Wiesbaden, 2000 . 
[OT08] L. G. Oversteegen and E. D. Tymchatyn, Extending isotopies of planar continua, arXiv:0811.0364v1.

[Pom92] Ch. Pommerenke, Boundary Behaviour of Conformal Maps, Springer, 1992.

[Rog98] J. T. Rogers, Jr., Diophantine conditions imply critical points on the boundaries of Siegel disks of polynomials, Comm. Math. Phys. 195 (1998), 175-193.

[Sie68] K. Sieklucki, On a class of plane acyclic continua with the fixed point property, Fund. Math. 63 (1968), 257-278.

[Ste35] L. Sternbach, Problem 107 (1935), in: The Scottish Book: Mathematics from the Scottish Café, Birkhäuser, Boston, 1981, 1935.

[Thu85] W. Thurston, The combinatorics of iterated rational maps (1985), in: Complex Dynamics: Families and Friends, D. Schleicher (ed.), A. K. Peters, 2008, Wellesley, MA, 1-108.

[Why42] G. T. Whyburn, Analytic Topology, Colloq. Publ. 28, Amer. Math. Soc., Providence, RI, 1942.

Alexander Blokh, Lex Oversteegen

Department of Mathematics

University of Alabama at Birmingham

Birmingham, AL 35294-1170, U.S.A.

E-mail: ablokh@math.uab.edu

overstee@math.uab.edu

Received 30 January 2009;

in revised form 19 May 2009 